“C 2007 IEEE. Personal use of this material is permitted. Permission from IEEE must be obtained for all other uses, in any current or future media, including reprinting/republishing this material for advertising or promotional purposes, creating new collective works, for resale or redistribution to servers or lists, or reuse of any copyrighted component of this work in other works." 


\title{
Convergence and Consistency Analysis for Extended Kalman Filter based SLAM
}

\author{
Shoudong Huang, Gamini Dissanayake \\ ARC Centre of Excellence for Autonomous Systems (CAS) \\ Faculty of Engineering \\ University of Technology, Sydney, Australia \\ Email: \{sdhuang,gdissa\}@eng.uts.edu.au \\ April 5, 2006
}

\begin{abstract}
This paper investigates the convergence properties and consistency of Extended Kalman Filter (EKF) based simultaneous localization and mapping (SLAM) algorithms. Proofs of convergence are provided for the nonlinear two-dimensional SLAM problem with point landmarks observed using a rangeand-bearing sensor. It is shown that the robot orientation uncertainty at the instant when landmarks are first observed has a significant effect on the limit and/or the lower bound of the uncertainties of the landmark position estimates. This paper also provides some insights to the inconsistencies of EKF based SLAM that have been recently observed. The fundamental cause of EKF SLAM inconsistency for two basic scenarios are clearly stated and associated theoretical proofs are provided.
\end{abstract}

Index Terms-Simultaneous localization and mapping (SLAM), Extended Kalman Filter, Extended Information Filter, Convergence, Inconsistency.

\section{INTRODUCTION}

Simultaneous Localization and Mapping (SLAM) is the process of building a map of an environment while concurrently generating an estimate for the pose of the robot. Many different techniques have been developed to solve the SLAM problem (see [1] and the references therein). However, the use of an Extended Kalman Filter (EKF) to estimate a state vector containing both the robot pose (including position and orientation) and the landmark locations (e.g. [2]) remains one of the most popular strategies for solving SLAM.

While there have been numerous implementations, only very few analytical results on the convergence and essential properties of the EKF SLAM algorithm are available. Dissanayake $e t$ al. provided convergence properties of SLAM and lower bound on the position uncertainty [2]. These results were extended to multi-robots SLAM in [3]. Kim [4] provided some further analysis on the asymptotic behavior for the one dimensional SLAM problem. All the proofs presented in the literature ([2][6]), however, only deals with simple linear formulations of the SLAM problem.

Almost all practical SLAM implementations need to deal with nonlinear process and observation models. The results due to [2] are intuitive and many early experiments and computer simulations appear to confirm that the properties of the linear solution extends to practical nonlinear problems. In the past few years, a number of researchers have demonstrated that the lower bound for the map accuracy presented in [2] is violated and the EKF SLAM produces inconsistent estimates due to errors introduced during the linearization process [7][8][9][10][11]. While some explanation of this phenomena has been reported, mainly through Monte-Carlo simulations, a thorough theoretical analysis of the nonlinear SLAM problem is not yet available.

This paper provides both the key convergence properties and the explicit formulas for the covariance matrices for some basic scenarios in the nonlinear two-dimensional EKF SLAM problem with point landmarks observed using a range-andbearing sensor. Some insights to, and theoretical proofs of the EKF SLAM inconsistencies are also given. The results in this paper demonstrate that:

- Most of the convergence properties in [2] are still true for the nonlinear case provided that the Jacobians used in the EKF equations are evaluated at the true states.

- The main reasons for inconsistency in EKF SLAM are due to (i) the violation of some fundamental constraints governing the relationship between various Jacobians when they are evaluated at the current state estimate, and (ii) the use of relative location information from robot to landmarks to update the absolute robot and landmark location estimates.

- The robot orientation uncertainty plays an important role in both the EKF SLAM convergence and the possible inconsistency. In the limit, the inconsistency of EKF SLAM may cause the variance of the robot orientation estimate to be incorrectly reduced to zero.

The paper is organized as follows. In Section II, the EKF SLAM algorithm is restated in a form more suitable for theoretical analysis. In Section III, some key convergence properties are proved. The theoretical explanations of the EKF SLAM inconsistency are given in Section IV. Section V provides some discussions on related work and further research topics. Section VI concludes the paper. Most of the proofs and relevant background material are given in Appendices. ${ }^{1}$

\section{RESTATEMENT OF THE EKF SLAM ALGORITHM}

In this section, the EKF SLAM algorithm is restated using slightly different notations and formulas in order to clearly

\footnotetext{
${ }^{1}$ Details of the proofs omitted due to space constraints are available from the first author.
} 
state and prove the results in this paper.

\section{A. State vector in $2 D$ EKF SLAM}

The state vector is denoted as ${ }^{2}$

$$
X=\left(\phi, X_{r}, X_{1}, \cdots, X_{N}\right),
$$

where $\phi$ is the robot orientation, $X_{r}=\left(x_{r}, y_{r}\right)$ is the robot position, $X_{1}=\left(x_{1}, y_{1}\right), \cdots, X_{N}=\left(x_{N}, y_{N}\right)$ are the positions of the $N$ point-landmarks. Note that the robot orientation $\phi$ is separated from the robot position because it plays a crucial role in the convergence and consistency analysis.

\section{B. Prediction}

1) Process Model: The robot process model considered in this paper is

$$
\left[\begin{array}{c}
\phi(k+1) \\
x_{r}(k+1) \\
y_{r}(k+1)
\end{array}\right]=\left[\begin{array}{c}
\phi(k)+f_{\phi}(\gamma(k), v(k), \delta \gamma, \delta v) \\
x_{r}(k)+(v(k)+\delta v) T \cos [\phi(k)] \\
y_{r}(k)+(v(k)+\delta v) T \sin [\phi(k)]
\end{array}\right],
$$

and is denoted as

$$
\left[\begin{array}{c}
\phi(k+1) \\
X_{r}(k+1)
\end{array}\right]=f\left(\phi(k), X_{r}(k), \gamma(k), v(k), \delta \gamma, \delta v\right)
$$

where $v, \gamma$ are the 'controls', $\delta v, \delta \gamma$ are zero-mean Gaussian noise on $v, \gamma . T$ is the time interval of one movement step. The explicit formula of function $f_{\phi}$ depends on the particular robot. Two examples of this general model are given below.

Example 1 A simple discrete-time robot motion model

$$
\begin{aligned}
& \phi(k+1)=\phi(k)+(\gamma(k)+\delta \gamma) T \\
& x_{r}(k+1)=x_{r}(k)+(v(k)+\delta v) T \cos [\phi(k)] \\
& y_{r}(k+1)=y_{r}(k)+(v(k)+\delta v) T \sin [\phi(k)]
\end{aligned}
$$

which can be obtained from a direct discretization of the unicycle model (e.g. [10])

$$
\begin{aligned}
& \dot{\phi}=\gamma \\
& \dot{x}_{r}=v \cos \phi \\
& \dot{y}_{r}=v \sin \phi
\end{aligned}
$$

where $v$ is the velocity and $\gamma$ is the turning rate.

Example 2 A car-like vehicle model (e.g. [2])

$$
\begin{aligned}
& \phi(k+1)=\phi(k)+\frac{(v(k)+\delta v) T \tan (\gamma(k)+\delta \gamma)}{L} \\
& x_{r}(k+1)=x_{r}(k)+(v(k)+\delta v) T \cos [\phi(k)] \\
& y_{r}(k+1)=y_{r}(k)+(v(k)+\delta v) T \sin [\phi(k)]
\end{aligned}
$$

where $v$ is the velocity and $\gamma$ is the steering angle, $L$ is the wheel-base of the vehicle.

The process model of landmarks (assumed stationary) is

$$
X_{i}(k+1)=X_{i}(k), \quad i=1, \cdots, N .
$$

Thus, the process model of the whole system is

$$
X(k+1)=F(X(k), \gamma(k), v(k), \delta \gamma, \delta v),
$$

where $F$ is the function combining (2) and (6).

\footnotetext{
${ }^{2}$ To simplify the notation, the vector transpose operator is omitted. For example, $X, X_{r}, X_{1}, \cdots, X_{N}$ are all column vectors and the rigorous notation should be $X=\left(\phi, X_{r}^{T}, X_{1}^{T}, \cdots, X_{N}^{T}\right)^{T}$.
}

2) Prediction: Suppose at time $k$, after the update, the estimate of the state vector is

$$
\hat{X}(k \mid k)=\left(\hat{\phi}(k), \hat{X}_{r}(k), \hat{X}_{1}, \cdots, \hat{X}_{N}\right),
$$

and the covariance matrix of the estimation error is $P(k \mid k)$. The prediction step is given by

$$
\begin{aligned}
\hat{X}(k+1 \mid k) & =F(\hat{X}(k \mid k), \gamma(k), v(k), 0,0), \\
P(k+1 \mid k) & =\nabla F_{\phi X_{r} X} P(k \mid k) \nabla F_{\phi X_{r} X}^{T}+\nabla F_{\gamma v} \Sigma \nabla F_{\gamma v}^{T},
\end{aligned}
$$

where $\Sigma$ is the covariance of the control noise $(\delta \gamma, \delta v)$, and $\nabla F_{\phi X_{r} X}, \nabla F_{\gamma v}$ are given by ${ }^{3}$

$$
\nabla F_{\phi X_{r} X}=\left[\begin{array}{cc}
\nabla f_{\phi X_{r}} & 0 \\
0 & I
\end{array}\right], \nabla F_{\gamma v}=\left[\begin{array}{c}
\nabla f_{\gamma v} \\
0
\end{array}\right] .
$$

Here $\nabla f_{\phi X_{r}}$ and $\nabla f_{\gamma v}$ are Jacobians of $f$ in (2) with respect to the robot pose $\left(\phi, X_{r}\right)$ and the control noise $(\delta \gamma, \delta v)$, respectively, evaluated at the current estimate $\hat{X}(k \mid k)$.

For the system described by equation (2), the Jacobian with respect to the robot pose is

$$
\nabla f_{\phi X_{r}}=\left[\begin{array}{ccc}
1 & 0 & 0 \\
-v T \sin \phi & 1 & 0 \\
v T \cos \phi & 0 & 1
\end{array}\right] .
$$

The Jacobian with respect to the controls, $\nabla f_{\gamma v}$, depends on the detailed formula of function $f_{\phi}$ in (2).

\section{Update}

1) Measurement Model: At time $k+1$, the measurement of $i$-th landmark, obtained using sensor on board the robot, is given by range $r_{i}$ and bearing $\theta_{i}$,

$$
\begin{aligned}
r_{i} & =\sqrt{\left(y_{i}-y_{r}(k+1)\right)^{2}+\left(x_{i}-x_{r}(k+1)\right)^{2}}+w_{r_{i}} \\
\theta_{i} & =\arctan \left(\frac{y_{i}-y_{r}(k+1)}{x_{i}-x_{r}(k+1)}\right)-\phi(k+1)+w_{\theta_{i}}
\end{aligned}
$$

where $w_{r_{i}}$ and $w_{\theta_{i}}$ are the noise on the measurements.

The observation model can be written in the general form

$$
z_{i}(k+1)=\left[\begin{array}{c}
r_{i} \\
\theta_{i}
\end{array}\right]=H_{i}(X(k+1))+w_{r_{i} \theta_{i}} .
$$

The noise $w_{r_{i} \theta_{i}}$ is assumed to be Gaussian with zero-mean and covariance matrix $R_{r_{i} \theta_{i}}$.

2) Update: Equation to update the covariance matrix can be written in the information form ([12]) as follows.

$$
\begin{aligned}
\Omega(k+1 \mid k) & =P(k+1 \mid k)^{-1}, \\
\Omega(k+1 \mid k+1) & =\Omega(k+1 \mid k)+\Omega_{\text {new }}, \\
P(k+1 \mid k+1) & =\Omega(k+1 \mid k+1)^{-1},
\end{aligned}
$$

where $\Omega(\cdot)$ is the information matrix, $\Omega_{\text {new }}$ is the new information obtained from the observation given by

$$
\Omega_{\text {new }}=\nabla H_{i}^{T} R_{r_{i} \theta_{i}}^{-1} \nabla H_{i}
$$

and $\nabla H_{i}$ is the Jacobian of function $H_{i}$ evaluated at the current estimate $\hat{X}(k+1 \mid k)$.

The estimate of the state vector can now be updated using

$$
\hat{X}(k+1 \mid k+1)=\hat{X}(k+1 \mid k)+W(k+1) \mu(k+1)
$$

\footnotetext{
${ }^{3}$ In this paper, $I$ and 0 always denote the identity matrix and a zero matrix with an appropriate dimension, respectively.
} 
where

$$
\begin{aligned}
\mu(k+1) & =z_{i}(k+1)-H_{i}(\hat{X}(k+1 \mid k)) \\
W(k+1) & =P(k+1 \mid k) \nabla H_{i}^{T} S^{-1}(k+1)
\end{aligned}
$$

and

$$
S(k+1)=R_{r_{i} \theta_{i}}+\nabla H_{i} P(k+1 \mid k) \nabla H_{i}^{T} .
$$

Remark 2.1: Using (13), (14) and the matrix inversion lemma (see equation (99) in Appendix B),

$$
\begin{aligned}
P(k+1 \mid k+1)= & P(k+1 \mid k)-P(k+1 \mid k) \nabla H_{i}^{T} \\
& \cdot\left(R_{r_{i} \theta_{i}}+\nabla H_{i} P(k+1 \mid k) \nabla H_{i}^{T}\right)^{-1} \\
& \cdot \nabla H_{i} P(k+1 \mid k),
\end{aligned}
$$

which is the typical EKF update formula.

The Jacobian of the measurement function $H_{i}$ is

$$
\nabla H_{i}=\left[\begin{array}{rrrrr}
0 & -\frac{d x}{r} & -\frac{d y}{r} & \frac{d x}{r} & \frac{d y}{r} \\
-1 & \frac{d y}{r^{2}} & -\frac{d x}{r^{2}} & -\frac{d y}{r^{2}} & \frac{d x}{r^{2}}
\end{array}\right]
$$

where

$$
\begin{aligned}
d x & =x_{i}-x_{r}(k+1) \\
d y & =y_{i}-y_{r}(k+1) \\
r & =\sqrt{d x^{2}+d y^{2}} .
\end{aligned}
$$

Note that, in the above, all the columns corresponding to landmarks that are not currently being observed have been ignored.

\section{Convergence Properties of EKF SLAM}

This section proves some convergence results for $2 \mathrm{D}$ nonlinear EKF SLAM. The first result is the monotonically decreasing property which is the same as Theorem 1 in [2].

Theorem 3.1: The determinant of any submatrix of the map covariance matrix decreases monotonically as successive observations are made.

Proof: This result can be proved in a similar way to that of Theorem 1 in [2]. The only difference is that the Jacobians instead of the state transition matrix and observation matrices will be used in the proof. The key point of the proof is "In the prediction step, the covariance matrix of the map does not change; in the update step, the whole covariance matrix is non-increasing". The details of the proof are omitted.

For 2D nonlinear EKF SLAM, general expressions for the covariance matrices evolution can not be obtained. Therefore, two basic scenarios are considered in the following: (1) the robot is stationary and observes new landmarks many times, and (2) the robot then moves but only observes the same landmarks.

Suppose the robot starts at point $\mathbf{A}$, the initial uncertainty of the robot pose is expressed by the covariance matrix

$$
P_{0}=\left[\begin{array}{cc}
p_{\phi} & \mathbf{p}_{x y \phi}^{T} \\
\mathbf{p}_{x y \phi} & P_{x y}
\end{array}\right]
$$

where $p_{\phi}$ is a scalar and $P_{x y}$ is a $2 \times 2$ matrix.

The initial information matrix is denoted as

$$
\Omega_{0}=P_{0}^{-1}=\left[\begin{array}{cc}
i_{\phi} & \mathbf{b}^{T} \\
\mathbf{b} & \Omega_{x y}
\end{array}\right] .
$$

\section{A. Scenario 1 - robot stationary}

Consider the scenario that the robot is stationary at point $\mathbf{A}$ and makes $n$ observations.

1) Observe one landmark: First assume that the robot can only observe one new landmark - landmark $m$. The Jacobian in (19) evaluated at the true landmark position $\left(x_{m}, y_{m}\right)$ and the true robot position $\left(x_{A}, y_{A}\right)$ is denoted as ${ }^{4}$

$$
\nabla H_{A}=\left[\begin{array}{lll}
-\mathbf{e} & -A & A
\end{array}\right],
$$

where

$$
\mathbf{e}=\left[\begin{array}{l}
0 \\
1
\end{array}\right], \quad A=\left[\begin{array}{rr}
\frac{d x_{A}}{r_{A}} & \frac{d y_{A}}{r_{A}} \\
-\frac{d y_{A}}{r_{A}^{2}} & \frac{d x_{A}}{r_{A}^{2}}
\end{array}\right],
$$

with

$$
\begin{aligned}
d x_{A} & =x_{m}-x_{A} \\
d y_{A} & =y_{m}-y_{A} \\
r_{A} & =\sqrt{d x_{A}^{2}+d y_{A}^{2}} .
\end{aligned}
$$

For convenience, further denote that

$$
A_{\mathbf{e}}=\left[\begin{array}{ll}
A^{-1} \mathbf{e} & I
\end{array}\right]
$$

where $I$ denotes $2 \times 2$ identity matrix (see footnote 3 ).

Theorem 3.2: If the robot is stationary and observes a new landmark $n$ times, the covariance matrix of the robot pose and the new landmark position estimates is

$$
P_{A_{\text {end }}}^{n}=\left[\begin{array}{cc}
P_{0} & P_{0} A_{\mathbf{e}}^{T} \\
A_{\mathbf{e}} P_{0} & A_{\mathbf{e}} P_{0} A_{\mathbf{e}}^{T}+\frac{A^{-1} R_{A} A^{-T}}{n}
\end{array}\right]
$$

where $P_{0}$ is the initial robot uncertainty given in (21), $A$ is defined in (24), $A_{\mathrm{e}}$ is defined in (26), and $R_{A}$ is the observation noise covariance matrix. In the limit when $n \rightarrow \infty$, the covariance matrix becomes

$$
P_{A_{\text {end }}}^{\infty}=\left[\begin{array}{cc}
P_{0} & P_{0} A_{\mathbf{e}}^{T} \\
A_{\mathbf{e}} P_{0} & A_{\mathbf{e}} P_{0} A_{\mathbf{e}}^{T}
\end{array}\right]=\left[\begin{array}{c}
I \\
A_{\mathbf{e}}
\end{array}\right] P_{0}\left[\begin{array}{ll}
I & A_{\mathbf{e}}^{T}
\end{array}\right] .
$$

Proof: See Appendix A.

The following corollary can be obtained from Theorem 3.2.

Corollary 3.3: If the robot is stationary and observes a new landmark $n$ times, the robot uncertainty remains unchanged. The limit (lower bound) on the covariance matrix of the new landmark is

$$
P_{A_{m}}^{\infty}=A_{\mathbf{e}} P_{0} A_{\mathbf{e}}^{T},
$$

which is determined by the robot uncertainty $P_{0}$ and the Jacobian $\nabla H_{A}$. In the special case when the initial uncertainty of the robot orientation $p_{\phi}$ is $0, P_{A_{m}}^{\infty}$ is equal to the initial robot position uncertainty $P_{x y}$ in (21).

Proof: See Appendix A. $\square$

Remark 3.4: Theorem 3.2 and Corollary 3.3 can be regarded as the nonlinear version of Theorem 3 in [2]. Moreover, it is clear that the robot orientation uncertainty has a significant effect on the limit of the landmark uncertainty. "When the robot position is exactly known but its orientation is uncertain,

\footnotetext{
${ }^{4}$ For the theoretical convergence results, the Jacobians are always evaluated at the true states. In the real SLAM applications, the Jacobians have to be evaluated at the estimated states and this may cause inconsistency. A detailed analysis of this is given in Section IV.
} 
even if there is a perfect knowledge about the relative location between the landmark and the robot, it is still impossible to tell exactly where the true landmark position is". $\square$

Figures 1(a) and 1(b) show that the initial robot orientation uncertainty has a significant effect on the landmark estimation accuracy. In Figure 1(a), the initial uncertainty of the robot pose is $P_{0}=\operatorname{diag}(0.03,1,1)$. Because the robot orientation uncertainty is large (the standard deviation is 0.1732 radians $\approx 10$ degrees), in the limit, the uncertainty of the landmark position is much larger than the initial uncertainty of the robot position. In Figure 1(b), the initial robot pose uncertainty is $P_{0}=\operatorname{diag}(0.001,1,1)$. Because the robot orientation uncertainty is very small (the standard deviation is 0.0316 radians $\approx 1.8$ degrees $)$, in the limit, the uncertainty of the landmark position is very close to the initial uncertainty of the robot position.

2) Observe two landmarks: Suppose the robot can observe two new landmarks (landmark $m$ and landmark $\bar{m}$ ) at point $\mathbf{A}$, then the dimension of the observation function in (12) is four (two ranges and two bearings), the Jacobian can be denoted as:

$$
\nabla \hat{H}_{A}=\left[\begin{array}{cccc}
-\mathbf{e} & -A & A & 0 \\
-\mathbf{e} & -\bar{A} & 0 & \bar{A}
\end{array}\right]
$$

where $\bar{A}$ is similar to $A$ in (24) but defined for landmark $\bar{m}$.

Similar to (26), denote

$$
\bar{A}_{\mathbf{e}}=\left[\begin{array}{ll}
\bar{A}^{-1} \mathbf{e} & I]
\end{array} .\right.
$$

The following theorem and corollary can now be obtained. The proofs are similar to that of Theorem 3.2 and Corollary 3.3 and are omitted here.

Theorem 3.5: If the robot is stationary and observes two new landmarks $n$ times, the covariance matrix of the robot pose and the two new landmark position estimates is

$$
\hat{P}_{A_{\text {end }}}^{n}=\left[\begin{array}{ccc}
P_{0} & P_{0} A_{\mathbf{e}}^{T} & P_{0} \bar{A}_{\mathbf{e}}^{T} \\
A_{\mathbf{e}} P_{0} & P_{A_{m}}^{n} & A_{\mathbf{e}} P_{0} \bar{A}_{\mathbf{e}}^{T} \\
\bar{A}_{\mathbf{e}} P_{0} & \bar{A}_{\mathbf{e}} P_{0} A_{\mathbf{e}}^{T} & P_{\bar{A}_{\bar{m}}}^{n}
\end{array}\right]
$$

where

$$
\begin{aligned}
& P_{A_{m}}^{n}=A_{\mathbf{e}} P_{0} A_{\mathbf{e}}^{T}+\frac{A^{-1} R_{A} A^{-T}}{n}, \\
& P_{\bar{A}_{\bar{m}}^{n}}^{n}=\bar{A}_{\mathbf{e}} P_{0} \bar{A}_{\mathbf{e}}^{T}+\frac{\bar{A}^{-1} R_{\bar{A}} \bar{A}^{-T}}{n},
\end{aligned}
$$

and $R_{\bar{A}}$ is the observation noise covariance matrix for observing landmark $\bar{m}$. In the limit when $n \rightarrow \infty$, the whole covariance matrix is

$$
\begin{aligned}
\hat{P}_{A_{\text {end }}}^{\infty} & =\left[\begin{array}{ccc}
P_{0} & P_{0} A_{\mathbf{e}}^{T} & P_{0} \bar{A}_{\mathbf{e}}^{T} \\
A_{\mathbf{e}} P_{0} & A_{\mathbf{e}} P_{0} A_{\mathbf{e}}^{T} & A_{\mathbf{e}} P_{0} \bar{A}_{\mathbf{e}}^{T} \\
\bar{A}_{\mathbf{e}} P_{0} & \bar{A}_{\mathbf{e}} P_{0} A_{\mathbf{e}}^{T} & \bar{A}_{\mathbf{e}} P_{0} \bar{A}_{\mathbf{e}}^{T}
\end{array}\right] \\
& =\left[\begin{array}{c}
I \\
A_{\mathbf{e}} \\
\bar{A}_{\mathbf{e}}
\end{array}\right] P_{0}\left[\begin{array}{lll}
I & A_{\mathbf{e}}^{T} & \bar{A}_{\mathbf{e}}^{T}
\end{array}\right] .
\end{aligned}
$$

Corollary 3.6: If the robot is stationary and observes two new landmarks $n$ times, the robot uncertainty remains unchanged. The limit (lower bound) of the covariance matrix associated with the two new landmarks is

$$
P_{A_{m \bar{m}}}^{\infty}=\left[\begin{array}{cc}
A_{\mathbf{e}} P_{0} A_{\mathbf{e}}^{T} & A_{\mathbf{e}} P_{0} \bar{A}_{\mathbf{e}}^{T} \\
\bar{A}_{\mathbf{e}} P_{0} A_{\mathbf{e}}^{T} & \bar{A}_{\mathbf{e}} P_{0} \bar{A}_{\mathbf{e}}^{T}
\end{array}\right] .
$$

In the special case when the initial uncertainty of the robot orientation $p_{\phi}=0$, the limit $P_{A_{m \bar{m}}}^{\infty}=\left[\begin{array}{cc}P_{x y} & P_{x y} \\ P_{x y} & P_{x y}\end{array}\right]$.

Remark 3.7: Theorem 3.5 and Corollary 3.6 are the analogue of Theorem 2 in [2]. However, because $A_{\mathbf{e}} \neq \bar{A}_{\mathbf{e}}$, $A_{\mathbf{e}} P_{0} A_{\mathbf{e}}^{T} \neq \bar{A}_{\mathbf{e}} P_{0} \bar{A}_{\mathbf{e}}^{T}$ when $p_{\phi} \neq 0$. This means that the limits of the uncertainties of the two landmarks are different when the robot orientation uncertainty is not zero. This is different from the linear results proved in [2], where the uncertainties of all the landmarks (with similar landmark types) are the same. This result is due to the nonlinearity of the observation function, which makes the Jacobians to be different when evaluated at locations of different landmarks.

Figure 2(a) shows that the difference between the uncertainties of the two landmarks is large when the robot orientation uncertainty $p_{\phi}$ is large $\left(p_{\phi}\right.$ is the same as that in Figure 1(a)). Figure 2(b) shows that the difference is very small when the initial robot orientation uncertainty $p_{\phi}$ is small $\left(p_{\phi}\right.$ is the same as that in Figure 1(b)).

\section{B. Scenario 2 - robot moves}

Consider the scenario that the robot first remains stationary at point $\mathbf{A}$ and makes observations $n \rightarrow \infty$ times. Then the robot moves to another observation point $\mathbf{B}$ in one time step, and observes the same landmarks $l$ times.

1) Observe one landmark: First assume that the robot can only observe one new landmark (at points $\mathbf{A}$ and B) landmark $m$. The Jacobian in (19) evaluated at point $\mathbf{B}$ and the true position of landmark $m$ is denoted as

$$
\nabla H_{B}=\left[\begin{array}{lll}
-\mathbf{e} & -B & B
\end{array}\right],
$$

where $B$ is similar to $A$ in (24) but defined for the robot pose at point B. Similar to (26), denote

$$
B_{\mathbf{e}}=\left[B^{-1} \mathbf{e} \quad I\right] \text {. }
$$

The following lemma gives the relationship between the Jacobians at point $\mathbf{A}$ and point $\mathbf{B}$.

Lemma 3.8: The relationship between the Jacobians at point $A$ and point $B$ is

$$
A_{\mathbf{e}}=B_{\mathbf{e}} \nabla f_{\phi X_{r}}^{A},
$$

where $\nabla f_{\phi X_{r}}^{A}$ is the Jacobian of $f$ in (2) with respect to the robot orientation and position (see equation (10)), evaluated at the robot pose $A$ and the associated control values.

Proof: See Appendix A. $\square$

The relationship given in Lemma 3.8 plays an important role in deriving the following convergence results. Furthermore, it will be shown in Theorem 4.2 in Section IV that the violation of this relationship may cause inconsistency in EKF SLAM.

Theorem 3.9: If the robot first remains stationary at point $A$ and observes one new landmark $n \rightarrow \infty$ times before it moves to point $B$ and observes the same landmark $l$ times, then the final covariance matrix is

$$
P_{B_{\text {end }}}^{l}=P_{B_{\text {start }}}^{0}+P_{B}^{l}
$$




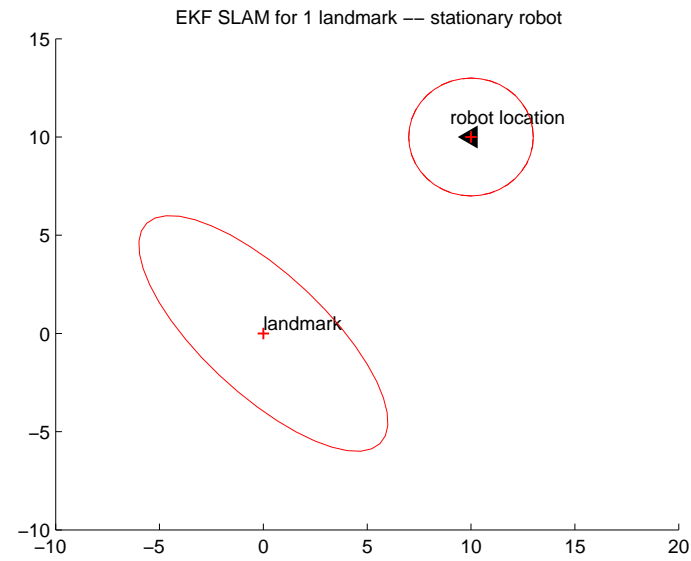

(a) Initial robot orientation uncertainty is large

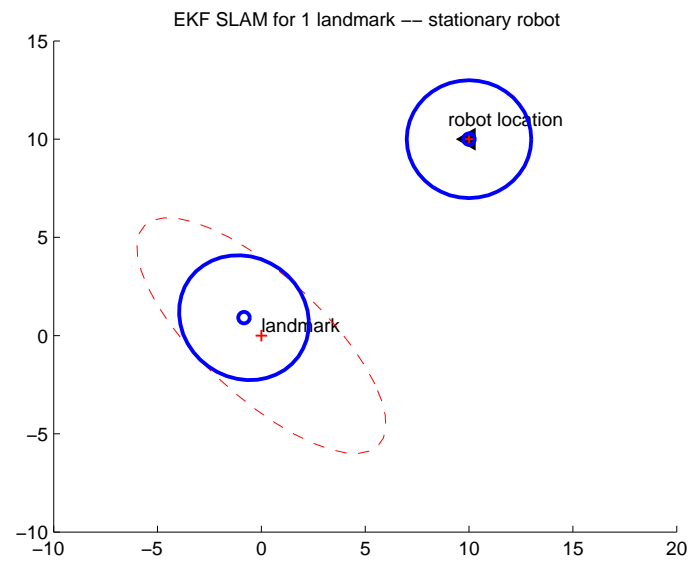

(c) Inconsistency of EKF SLAM

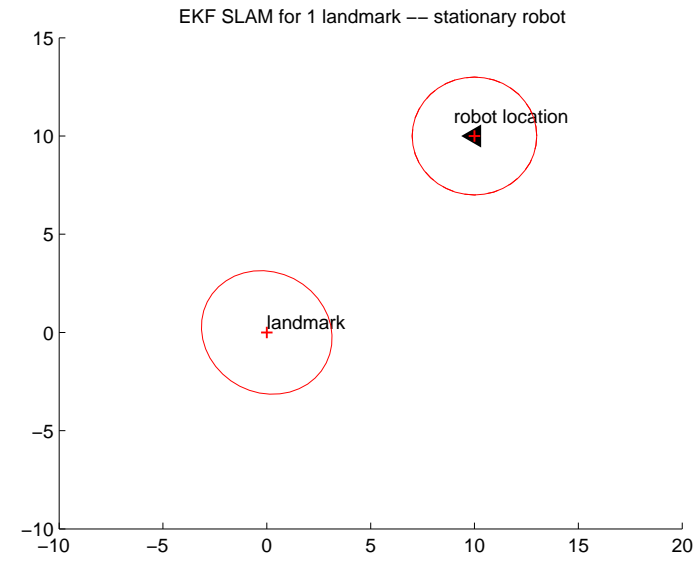

(b) Initial robot orientation uncertainty is small

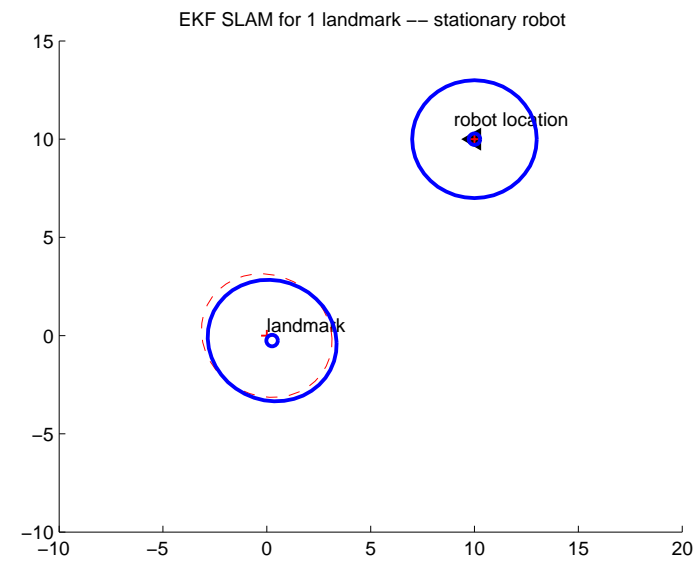

(d) Inconsistency can be neglected when initial robot orientation uncertainty is small

Fig. 1. The limits of landmark uncertainty when the robot is stationary and observes the landmark $n \rightarrow \infty$ times (see Theorem 3.2 , Corollary 3.3 and Theorem 4.1): In Figure 1(a) and Figure 1(c), the initial uncertainty of the robot pose is $P_{0}=\operatorname{diag}(0.03,1,1)$. In Figure 1(b) and Figure 1(d), the initial robot pose uncertainty is $P_{0}=\operatorname{diag}(0.001,1,1)$. For Figure $1(\mathrm{a})$ and Figure $1(\mathrm{~b})$, the Jacobians are evaluated at the true robot and landmark locations. In Figures 1(c) and 1(d), the solid ellipses are the limit of the uncertainties when the Jacobians are evaluated at the updated state estimate at each update step.

where

$$
\begin{aligned}
& P_{B_{\text {start }}}^{0}=\left[\begin{array}{cc}
\nabla f_{\phi X_{r}}^{A} P_{0}\left(\nabla f_{\phi X_{r}}^{A}\right)^{T} & \nabla f_{\phi X_{r}}^{A} P_{0} A_{\mathbf{e}}^{T} \\
A_{\mathbf{e}} P_{0}\left(\nabla f_{\phi X_{r}}^{A}\right)^{T} & A_{\mathbf{e}} P_{0} A_{\mathbf{e}}^{T}
\end{array}\right] \\
&= {\left[\begin{array}{cc}
\nabla f_{\phi X_{r}}^{A} & 0 \\
0 & I
\end{array}\right] P_{A_{\text {end }}}^{\infty}\left[\begin{array}{cc}
\left(\nabla f_{\phi X_{r}}^{A}\right)^{T} & 0 \\
0 & I
\end{array}\right] } \\
& P_{B}^{l}=\left[\begin{array}{cc}
\nabla f_{\gamma v}^{A} \Sigma_{B}^{l}\left(\nabla f_{\gamma v}^{A}\right)^{T} & 0 \\
0 & 0
\end{array}\right]
\end{aligned}
$$

with

$$
\Sigma_{B}^{l}=\left[\Sigma^{-1}+l H_{A B}^{T} R_{B}^{-1} H_{A B}\right]^{-1} \geq 0
$$

and

$$
H_{A B}=\left[\begin{array}{ll}
\mathbf{e} & B
\end{array}\right] \nabla f_{\gamma v}^{A} .
$$

Furthermore, if the matrix $H_{A B}^{T} R_{B}^{-1} H_{A B}$ is invertible ${ }^{5}$, then the matrix $P_{B}^{l} \rightarrow 0$ when $l \rightarrow \infty$. Here $P_{A_{\text {end }}}^{\infty}$ is defined in (28), $R_{B}$ is the covariance matrix of the observation noise at point $\mathbf{B}, \nabla f_{\phi X_{r}}^{A}$ and $\nabla f_{\gamma v}^{A}$ are Jacobians of

\footnotetext{
${ }^{5}$ This depends on the process model and the direction of the robot movement but this is true in most of the cases.
}

\section{function $f$ in (2) evaluated at point $A$ and the associated control values.}

\section{Proof: See Appendix A.}

By Theorem 3.9, the lower bound of the covariance matrix is $P_{B_{\text {start }}}^{0}$, which is the covariance matrix when the robot first reaches point $\mathbf{B}$ if there is no control noise in moving from $\mathbf{A}$ to $\mathbf{B}(\Sigma=0$ in $(8))$.

Figures 3(a), 3(b), 3(c), and 3(d) illustrate Theorem 3.9. The initial robot uncertainty is the same as that used for Figure 1(a). Figures 3(a) and 3(b) show the case when there is no control noise. Figure 3(a) shows the uncertainties after the prediction step and Figure 3(b) shows the uncertainties after the update using the observations at point $\mathbf{B}$. It can be seen that the observations at point $\mathbf{B}$ can not reduce the uncertainty of the robot and landmarks. Figures 3(c) and 3(d) show the case when control noise is present. In this case, the landmark uncertainty cannot be improved by the observation at point $\mathbf{B}$, while the uncertainty of the robot can be reduced to the same level as the case when there is no control noise. The limits 


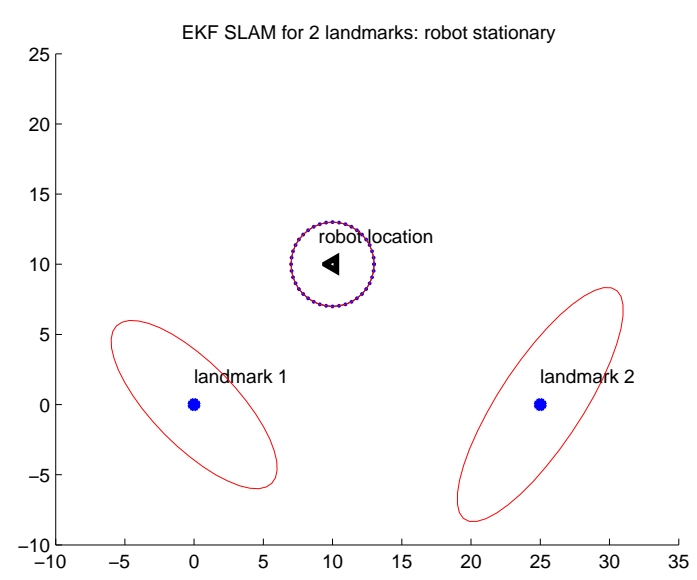

(a) Initial robot orientation uncertainty is large

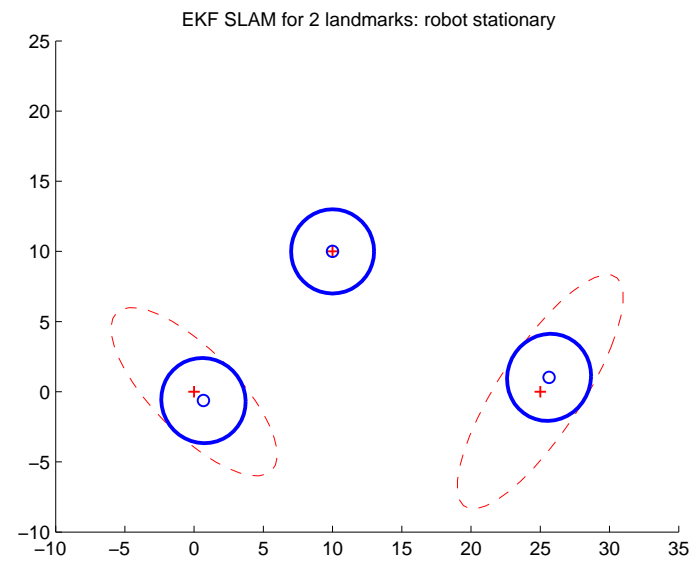

(c) Inconsistency of EKF SLAM for two landmarks

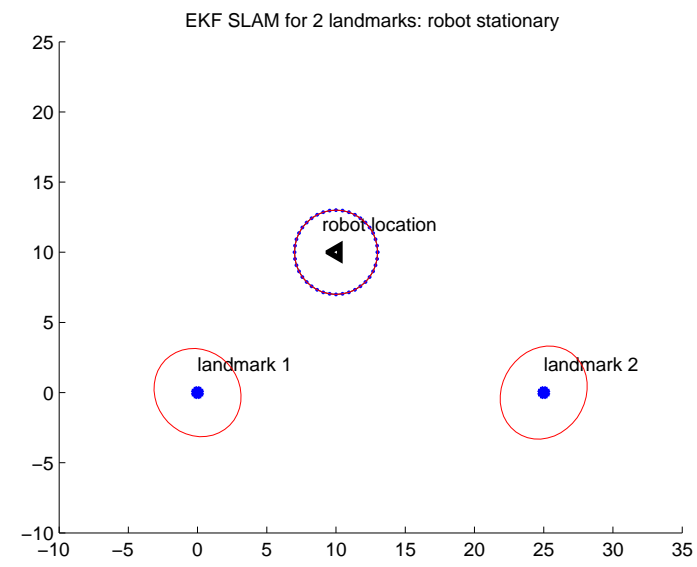

(b) Initial robot orientation uncertainty is small

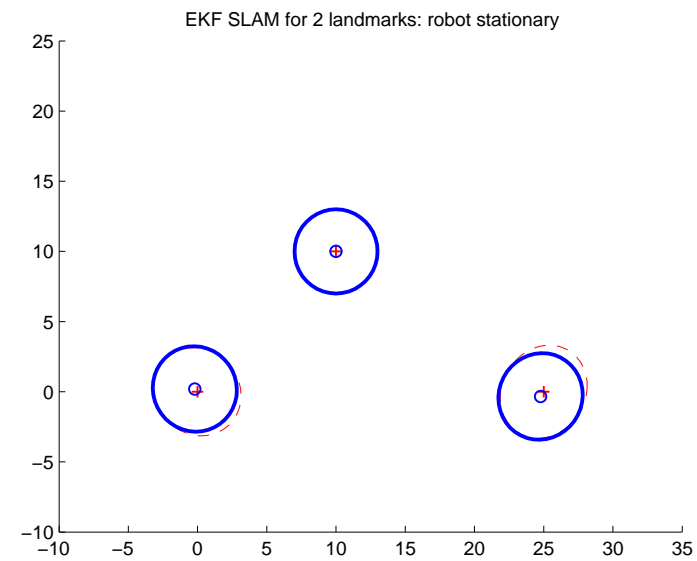

(d) Inconsistency can be neglected when initial robot orientation uncertainty is small

Fig. 2. The limits of the two landmark uncertainties when the robot is stationary and makes observation $n \rightarrow \infty$ times: Figure 2 (a) shows that the final uncertainties of the two landmarks are different. See Theorem 3.5, Corollary 3.6, Remark 3.7, Theorem 4.1 and the caption of Figure 1 for more explanations.

of the uncertainties are independent of the extent of sensor and control noises. The control noise only affect the robot uncertainty after the prediction in Figure 3(c). The sensor noise used are the same as those in Figure 1, the robot speed and the control noises (in Figures 3(c) and 3(d)) are deliberately enlarged, just to make the differences of the ellipses visible.

2) Observe two landmarks: Suppose the robot can observe two new landmarks (landmark $m$ and landmark $\bar{m}$ ) at points $\mathbf{A}$ and $\mathbf{B}$, then the dimension of the observation function in (12) is four (two ranges and two bearings), denote the corresponding Jacobians as $\nabla \hat{H}_{A}$ given in (30) and

$$
\nabla \hat{H}_{B}=\left[\begin{array}{llll}
-\mathbf{e} & -B & B & 0 \\
-\mathbf{e} & -\bar{B} & 0 & \bar{B}
\end{array}\right]
$$

Theorem 3.10: If the robot first remains stationary at point $A$ and observes two new landmarks $n \rightarrow \infty$ times before it moves to point $B$ and observes the same two landmarks $l$ times, then the final covariance matrix is

$$
\hat{P}_{B_{\text {end }}}^{l}=\hat{P}_{B_{\text {start }}}^{0}+\hat{P}_{B}^{l}
$$

where

$$
\begin{gathered}
\hat{P}_{B_{\text {start }}^{0}=}\left[\begin{array}{ccc}
\nabla f_{\phi X_{r}}^{A} & 0 & 0 \\
0 & I & 0 \\
0 & 0 & I
\end{array}\right] \hat{P}_{A_{\text {end }}}^{\infty} \\
\cdot\left[\begin{array}{ccc}
\left(\nabla f_{\phi X_{r}}^{A}\right)^{T} & 0 & 0 \\
0 & I & 0 \\
0 & 0 & I
\end{array}\right] \\
\hat{P}_{B}^{l}=\left[\begin{array}{ccc}
\nabla f_{\gamma v}^{A} \hat{\Sigma}_{B}^{l}\left(\nabla f_{\gamma v}^{A}\right)^{T} & 0 & 0 \\
0 & 0 & 0 \\
0 & 0 & 0
\end{array}\right],
\end{gathered}
$$

with

$$
\hat{\Sigma}_{B}^{l}=\left[\Sigma^{-1}+l\left(H_{A B}^{T} R_{B}^{-1} H_{A B}+H_{A \bar{B}}^{T} R_{\bar{B}}^{-1} H_{A \bar{B}}\right)\right]^{-1} \geq 0,
$$

and

$$
H_{A \bar{B}}=\left[\begin{array}{ll}
\mathbf{e} & \bar{B}
\end{array}\right] \nabla f_{\gamma v}^{A} .
$$

Furthermore, if the matrix $H_{A B}^{T} R_{B}^{-1} H_{A B}+H_{A \bar{B}}^{T} R_{\bar{B}}^{-1} H_{A \bar{B}}$ is invertible, then the matrix $\hat{P}_{B}^{l} \rightarrow 0$ when $l \rightarrow \infty$. Here $\hat{P}_{A_{\text {end }}}^{\infty}$ is defined in (34), $\nabla f_{\phi X_{r}}^{A}$ and $\nabla f_{\gamma v}^{A}$ are Jacobians of 

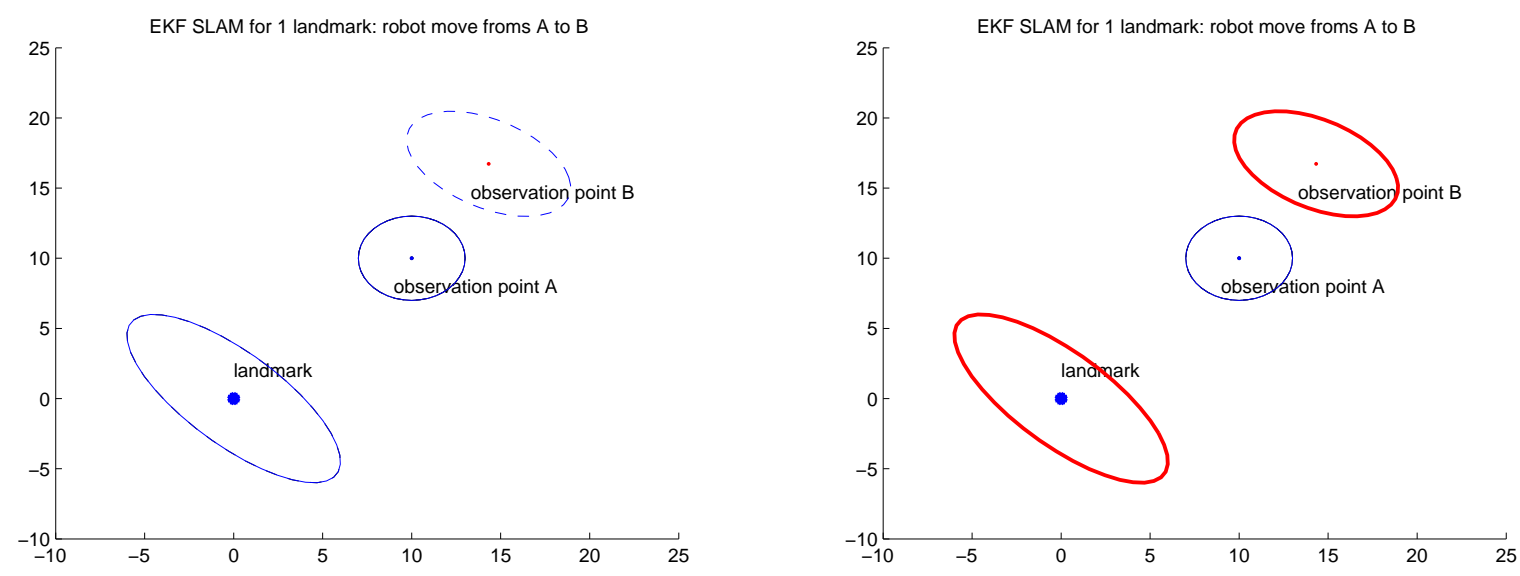

(a) no control noise - after prediction

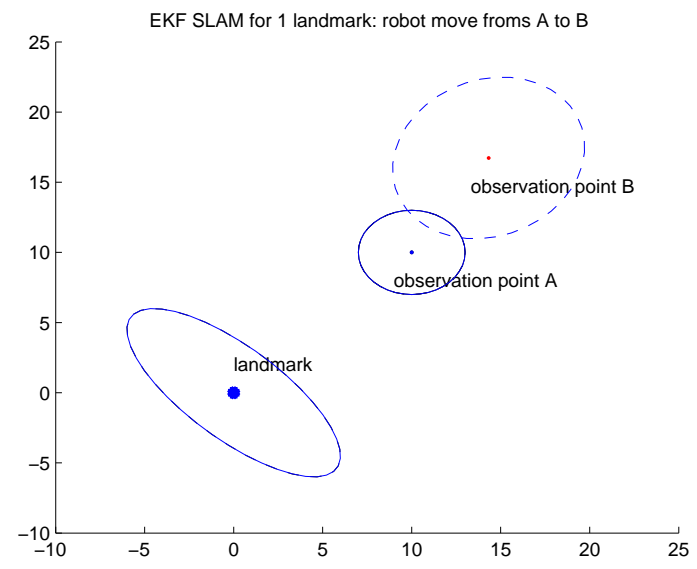

(b) no control noise - after update

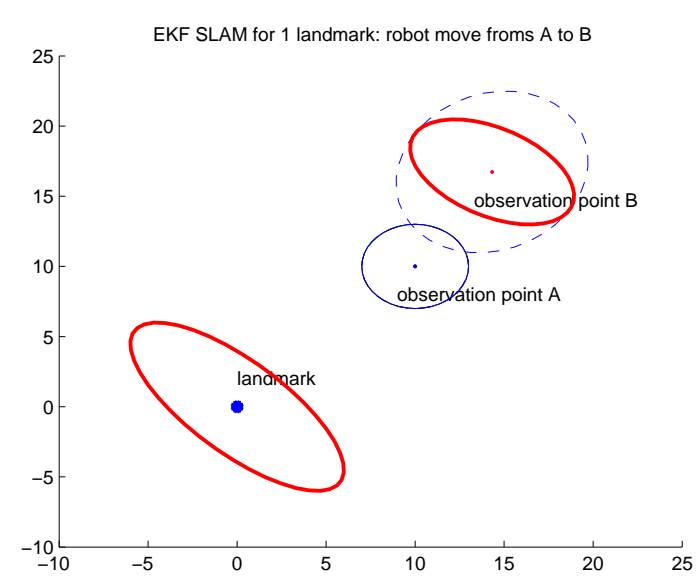

(c) with control noise - after prediction

(d) with control noise - after update

Fig. 3. The limits of the robot and landmark uncertainties when the robot first remains stationary at point $\mathbf{A}$ and makes observation $n \rightarrow \infty$ times and then moves to $\mathbf{B}$ and observes the same landmark $l \rightarrow \infty$ times (see Theorem 3.9)

function $f$ in (2) evaluated at point $A$ and the associated control values, $H_{A B}$ is defined in (43), and $R_{B}, R_{\bar{B}}$ are the covariance matrices of the observation noise at point B and for observing landmarks $m$ and $\bar{m}$, respectively.

Proof: The proof is similar to that of Theorem 3.9 and is omitted.

Remark 3.11: Theorems 3.9 and 3.10 show that the only effect of the observations made at point $\mathbf{B}$ is to reduce the additional robot uncertainty generated from the process noise. The observations made at point $\mathbf{B}$ cannot reduce the uncertainty of the landmark further if the robot had already observed the landmark many times at point A. Theorems 3.9 and 3.10 can be extended to the case when it takes more than one step to move from $\mathbf{A}$ to $\mathbf{B}$ such as $\mathbf{A} \rightarrow \mathbf{B}_{1} \rightarrow \mathbf{B}_{2} \rightarrow \ldots \rightarrow \mathbf{B}_{n} \rightarrow \mathbf{B}$. For example, for the one landmark case, the limit of the covariance matrix satisfies

$$
P_{B_{\text {end }}}^{\infty} \geq\left[\begin{array}{cc}
F_{A B} P_{0} F_{A B}^{T} & F_{A B} P_{0} A_{\mathbf{e}}^{T} \\
A_{\mathbf{e}} P_{0} F_{A B}^{T} & A_{\mathbf{e}} P_{0} A_{\mathbf{e}}^{T}
\end{array}\right],
$$

where

$$
F_{A B}=\nabla f_{\phi X_{r}}^{B_{n}} \cdots \nabla f_{\phi X_{r}}^{B_{1}} \nabla f_{\phi X_{r}}^{A}
$$

Figures 4(b) and 4(d) illustrate the results.

\section{CONSISTENCY OF EKF SLAM}

In all the theoretical convergence properties proved in the previous section, it is assumed that the Jacobians are evaluated at the true robot pose and the true landmark positions. In a real-life SLAM, the true locations of the robot and landmarks are not known, and the Jacobians have to be evaluated at the estimated values. This section provides a proof that this may result in over-confident (inconsistent) estimates.

\section{A. Why inconsistency can occur in the nonlinear EKF SLAM?}

A number of recent publications indicate that the key source of EKF SLAM inconsistency is the error introduced during the linearization process (e.g. [8][9]). While it is clear that linearization is an approximation which can introduce errors in to the estimation process, it is reasonable to expect that the incorrect estimate is likely to be either too optimistic (estimated uncertainty smaller than true uncertainty) or too pessimistic (estimated uncertainty larger than true uncertainty). However, the SLAM literature only reports estimator inconsistency as 
a result of optimistic estimates. No instances of pessimistic estimates during EKF SLAM has been reported. Why?

\section{B. An intuitive explanation}

In most cases, the measurement available for use in the SLAM algorithms is the relative location between the robot and landmarks, and the objective of the SLAM process is to estimate the absolute robot and landmark locations.

Suppose $x, y$ are two numbers and that two noisy measurements of $x-y$ are available:

$$
\begin{aligned}
& x-y \approx 99.8 \\
& x-y \approx 100.1
\end{aligned}
$$

Based on these measurements, although it is possible to say " $x-y$ is around $100 "$, nothing can be said about the true values of $x$ and/or $y$. However, if the measurement equation is non-linear, the linearized version of this equation may look like

$$
\begin{aligned}
& 1.01 x-y \approx 99.8 \\
& 0.99 x-y \approx 100.1
\end{aligned}
$$

From these two equations, the (approximate) absolute values of $x$ and $y$ can be calculated. Obviously, this outcome is too optimistic (inconsistent).

In the next two subsections, it will be shown that the mechanism that causes overconfident estimates in SLAM is similar to that presented above. Theoretical proofs are given for two basic scenarios.

\section{Scenario 1 - robot stationary}

In EKF SLAM, the observation innovation $(\mu(k+1)$ in (16)) is used to update the previous estimate $\hat{X}$. Through linearization, the innovation can be expressed as (see (23))

$$
\begin{aligned}
\mu & =z-H(\hat{X}) \\
& \approx H(X)-H(\hat{X}) \\
& \approx \nabla H_{A}(X-\hat{X}) \\
& =-\mathbf{e}(\phi-\hat{\phi})-A\left(X_{r}-\hat{X}_{r}\right)+A\left(X_{m}-\hat{X}_{m}\right),
\end{aligned}
$$

where $\hat{\phi}, \hat{X}_{r}$ and $\hat{X}_{m}$ are the estimates of the robot orientation, the robot position, and the landmark position, respectively. (54) is equivalent to

$$
\mathbf{e} \phi+A X_{r}-A X_{m} \approx-\mu+\mathbf{e} \hat{\phi}+A \hat{X}_{r}-A \hat{X}_{m} .
$$

Suppose the robot is stationary at point $\mathbf{A}$ and makes two consecutive observations to landmark $m-z_{1}$ and $z_{2}$. After the update using $z_{1}$, the estimates of the robot orientation, the robot position, and the landmark position will change from $\hat{\phi}, \hat{X}_{r}, \hat{X}_{m}$ to $\hat{\phi}^{1}, \hat{X}_{r}^{1}, \hat{X}_{m}^{1}$, thus the Jacobian will be evaluated at a different point in the state space when $z_{2}$ is used for the next update. The two innovations $\mu_{1}, \mu_{2}$ give

$$
\begin{aligned}
\mathbf{e} \phi+\tilde{A}_{1} X_{r}-\tilde{A}_{1} X_{m} & \approx-\mu_{1}+\mathbf{e} \hat{\phi}+\tilde{A}_{1} \hat{X}_{r}-\tilde{A}_{1} \hat{X}_{m}, \\
\mathbf{e} \phi+\tilde{A}_{2} X_{r}-\tilde{A}_{2} X_{m} & \approx-\mu_{2}+\mathbf{e} \hat{\phi}^{1}+\tilde{A}_{2} \hat{X}_{r}^{1}-\tilde{A}_{2} \hat{X}_{m}^{1},
\end{aligned}
$$

where $\tilde{A}_{1}, \tilde{A}_{2}$ are defined in a manner similar to (24) but computed at the estimated robot and landmark locations. Both
$\tilde{A}_{1}, \tilde{A}_{2}$ are non-singular matrices that are different but close to $A$.

The above two equations are equivalent to

$$
\begin{aligned}
\tilde{A}_{1}^{-1} \mathbf{e} \phi+X_{r}-X_{m} & \approx-\tilde{A}_{1}^{-1} \mu_{1}+\tilde{A}_{1}^{-1} \mathbf{e} \hat{\phi}+\hat{X}_{r}-\hat{X}_{m}, \\
\tilde{A}_{2}^{-1} \mathbf{e} \phi+X_{r}-X_{m} & \approx-\tilde{A}_{2}^{-1} \mu_{2}+\tilde{A}_{2}^{-1} \mathbf{e} \hat{\phi}^{1}+\hat{X}_{r}^{1}-\hat{X}_{m}^{1} .
\end{aligned}
$$

So

$$
\begin{aligned}
\left(\tilde{A}_{1}^{-1} \mathbf{e}-\tilde{A}_{2}^{-1} \mathbf{e}\right) \phi \approx & \tilde{A}_{2}^{-1} \mu_{2}-\tilde{A}_{1}^{-1} \mu_{1}+\hat{X}_{r}-\hat{X}_{m} \\
& +\tilde{A}_{1}^{-1} \mathbf{e} \hat{\phi}-\tilde{A}_{2}^{-1} \mathbf{e} \hat{\phi}^{1}-\hat{X}_{r}^{1}+\hat{X}_{m}^{1} .
\end{aligned}
$$

By the special structure of $\tilde{A}_{1}, \tilde{A}_{2}$ (see (24)), if $\tilde{A}_{1} \neq \tilde{A}_{2}$, then $\tilde{A}_{1}^{-1} \mathbf{e} \neq \tilde{A}_{2}^{-1} \mathbf{e}$ and equation (58) provides some information on the value of $\phi$. It is obvious that observing a single new landmark will not improve the knowledge of the robot orientation. Therefore, this apparent information on the robot orientation is incorrect and will result in overconfident estimates (inconsistency).

To examine the extent of the possible inconsistency, let the robot be stationary at point $\mathbf{A}$ and observe a new landmark $n$ times. Let the estimate be updated after each observation using Jacobians evaluated at the updated estimate at each time step. Denote the different Jacobians as

$$
\nabla H_{\tilde{A}_{j}}=\left[\begin{array}{lll}
-\mathbf{e} & -\tilde{A}_{j} & \tilde{A}_{j}
\end{array}\right], \quad 1 \leq j \leq n .
$$

Let $R_{A}$ denote the observation noise covariance matrix at point A, and define

$$
\begin{aligned}
w(n, A)= & n \mathbf{e}^{T} R_{A}^{-1} \mathbf{e}-\mathbf{e}^{T} R_{A}^{-1}\left(\sum_{j=1}^{n} \tilde{A}_{j}\right) \\
& \cdot\left(\sum_{j=1}^{n} \tilde{A}_{j}^{T} R_{A}^{-1} \tilde{A}_{j}\right)^{-1}\left(\sum_{j=1}^{n} \tilde{A}_{j}^{T}\right) R_{A}^{-1} \mathbf{e} .
\end{aligned}
$$

As before, suppose that the initial robot uncertainty is $P_{0}$ given by (21).

Theorem 4.1: In EKF SLAM, if the robot is stationary at point $\mathrm{A}$ and observes a new landmark $n$ times, the inconsistency occurs due to the fact that Jacobians are evaluated at different state estimates. The level of inconsistency is determined by the initial robot uncertainty $P_{0}$ and the $w(n, A)$ defined in (60). When $n \rightarrow \infty$, the inconsistency may cause the variance of the robot orientation estimate to be reduced to zero.

Proof: See Appendix A.

Figures 1(c), 1(d), 2(c), and 2(d) illustrate the results in Theorem 4.1. In Figure 1(c), the initial uncertainty of the robot pose is the same as that used in Figure 1(a), the solid ellipse is the limit of the landmark uncertainty when the Jacobian is evaluated at the updated state estimate at each update step. This figure is generated by performing 1000 updates assuming that the range and bearing measurements are corrupted by random Gaussian noise (the standard deviations of range and bearing noise are selected to be similar to that of a typical indoor laser scanner, $0.1 \mathrm{~m}$ and $1^{\circ}$, respectively). It can be seen that the uncertainty of the landmark is reduced far below the theoretical limit (dashed ellipse), demonstrating the inconsistency of EKF SLAM solution. In Figure 1(d), the initial uncertainty of the robot orientation is much smaller (the same as that used in Figure 1(b)). It can be seen that the extent 
of inconsistency is too small to be seen (the solid ellipse almost coincides with the dashed one).

\section{Scenario 2 - robot moves}

Consider the scenario that the robot observes a new landmark at point $\mathbf{A}$ and then moves to point $\mathbf{B}$ and makes an observation of the same landmark. Similar to (57), the two innovations $\mu_{A}, \mu_{B}$ give

$$
\begin{aligned}
\tilde{A}^{-1} \mathbf{e} \phi^{A}+X_{r}^{A}-X_{m} \approx & -\tilde{A}^{-1} \mu_{A}+\tilde{A}^{-1} \mathbf{e} \hat{\phi}^{A} \\
& +\hat{X}_{r}^{A}-\hat{X}_{m}^{A}, \\
\tilde{B}^{-1} \mathbf{e} \phi^{B}+X_{r}^{B}-X_{m} \approx & -\tilde{B}^{-1} \mu_{B}+\tilde{B}^{-1} \mathbf{e} \hat{\phi}^{B} \\
& +\hat{X}_{r}^{B}-\hat{X}_{m}^{B} .
\end{aligned}
$$

From the process model (2) with appropriate linearization,

$$
\begin{aligned}
\phi^{B} \approx & \phi^{A}+f_{\phi}(\hat{\gamma}, \hat{v}, 0,0) \\
X_{r}^{B} \approx & X_{r}^{A}+\left[\begin{array}{r}
\hat{v} T \cos \left(\hat{\phi}_{A}\right) \\
\hat{v} T \sin \left(\hat{\phi}_{A}\right)
\end{array}\right] \\
& +\left[\begin{array}{r}
-\hat{v} T \sin \left(\hat{\phi}_{A}\right) \\
\hat{v} T \cos \left(\hat{\phi}_{A}\right)
\end{array}\right]\left(\phi^{A}-\hat{\phi}_{A}\right) .
\end{aligned}
$$

Thus

$$
\begin{aligned}
& \left(\tilde{A}^{-1} \mathbf{e}-\tilde{B}^{-1} \mathbf{e}-\left[\begin{array}{r}
-\hat{v} T \sin \left(\hat{\phi}_{A}\right) \\
\hat{v} T \cos \left(\hat{\phi}_{A}\right)
\end{array}\right]\right) \phi^{A} \\
\approx & \tilde{B}^{-1} \mu_{B}-\tilde{A}^{-1} \mu_{A}+\tilde{A}^{-1} \mathbf{e} \hat{\phi}^{A}+\hat{X}_{r}^{A}-\hat{X}_{m}^{A} \\
& -\tilde{B}^{-1} \mathbf{e} \hat{\phi}^{B}-\hat{X}_{r}^{B}+\hat{X}_{m}^{B}+\tilde{B}^{-1} \mathbf{e} f_{\phi}(\hat{\gamma}, \hat{v}, 0,0) \\
+ & {\left[\begin{array}{c}
\hat{v} T \cos \left(\hat{\phi}_{A}\right) \\
\hat{v} T \sin \left(\hat{\phi}_{A}\right)
\end{array}\right]-\left[\begin{array}{r}
-\hat{v} T \sin \left(\hat{\phi}_{A}\right) \\
\hat{v} T \cos \left(\hat{\phi}_{A}\right)
\end{array}\right] \hat{\phi}_{A} . }
\end{aligned}
$$

If $\tilde{A}^{-1} \mathbf{e} \neq \tilde{B}^{-1} \mathbf{e}+\left[\begin{array}{r}-\hat{v} T \sin \left(\hat{\phi}_{A}\right) \\ \hat{v} T \cos \left(\hat{\phi}_{A}\right)\end{array}\right]$, then the above equation contains information on $\phi^{A}$, which is clearly incorrect as observations to a single landmark do not provide any knowledge about the robot orientation.

Note that $\tilde{A}^{-1} \mathbf{e}=\tilde{B}^{-1} \mathbf{e}+\left[\begin{array}{r}-\hat{v} T \sin \left(\hat{\phi}_{A}\right) \\ \hat{v} T \cos \left(\hat{\phi}_{A}\right)\end{array}\right]$ is actually the relationship proved in Lemma 3.8. Therefore, the following result can now be stated.

Theorem 4.2: When the robot observes the same landmark at two different points A and B, the EKF SLAM algorithm may provide inconsistent estimates due to the fact that the Jacobians evaluated at the estimated robot positions may violate the key relationship between the Jacobians as shown in Lemma 3.8.

Proof: See Appendix A.

Figures 4(a)-4(d) illustrate the extent of inconsistency under scenario 2. The robot first keeps still at point $\mathbf{A}$ and makes $n=10000$ observations. The initial robot uncertainty is the same as that used in Figure 1(a). The true Jacobians are used at point $\mathbf{A}$ to guarantee the consistency of the estimate before the robot moves. The robot then moves 500 steps to $\mathbf{B}$ and keeps observing the same landmark while moving. The thin/solid ellipses illustrate the estimate uncertainty after the observation at point $\mathbf{A}$. The dashed ellipses correspond to the uncertainties at the intermediate points (every 100 steps) while the thick/solid ellipses illustrate the final uncertainty. Figure 4(a) shows that the extent of inconsistency is quite significant when there is no control noise. Figure 4(b) shows the corresponding results where true Jacobians are used. Figure 4(c) shows the inconsistency when control noise is present. Figure 4(d) shows the corresponding results where true Jacobians are used. In this simulation, the sensor noise used were the same as that used in Figure 1, the control noise were chosen to be similar to that of Pioneer robots - standard deviations of velocity noise and turn rate noise are $0.02 \mathrm{~m} / \mathrm{s}$ and $3^{\circ} / \mathrm{s}$, respectively. The similarity between Figures 4(b) and 4(d) is due to the relatively small sensor noise where after the update, the uncertainty is almost the same as that obtained when there is no control noise (see Figure 3).

In the simulations presented in this paper, the magnitudes of the sensor noise and control noise were selected to be similar to those of a typical indoor-laser and Pioneer robots (except for the control noise in Figure 3). The effects of the sensor noise and control noise on the extent of inconsistency are complex and need further investigation. In general, larger noise may result in larger errors in the Jacobians but the amount of "wrong information" contained in (58) or (63) is also less when the noise are larger.

The inconsistency results in this paper only focus on the covariance matrices. The inconsistent mean estimate naturally results from the inconsistent covariance matrix because the Kalman gain in the subsequent step will be incorrect once the covariance matrix becomes inconsistent. See for example the means in Figures 1(c), 2(c), 4(a), 4(c).

\section{RELATED WORK AND DISCUSSION}

\section{A. Related work}

Consistency issue in mapping was recognized as a fundamental problem as early as 1986 when estimation-theoretic methods in robotic mapping became popular [13]. It took some time before it was realized that the correlations between landmarks are critical to guarantee convergence for SLAM [14]. An EKF SLAM algorithm that keeps all the correlations between robot pose and all the landmarks was described and some key convergence properties were proved in 2001 by Dissanayake et al. [2]. Since then, EKF SLAM has been regarded as a theoretically sound approach and has been used in many SLAM applications.

However, the convergence proofs given in [2] is only for linear case and it has been shown recently by a number of researchers that EKF SLAM can produce inconsistent (over-confident) estimations [7][8][9][10][11]. The theoretical analysis and the results presented in this paper further confirm this claim.

Frese [9] and Bailey et al. [11] pointed out that the robot orientation uncertainty is the main cause of the inconsistency in EKF SLAM. Although extensive simulation results are available to show that the inconsistency does exist, and almost all of the related papers point out that linearization is the cause of the inconsistency, the only theoretical explanation is given by [7]. This work, however, only deals with the case when the robot is stationary. 


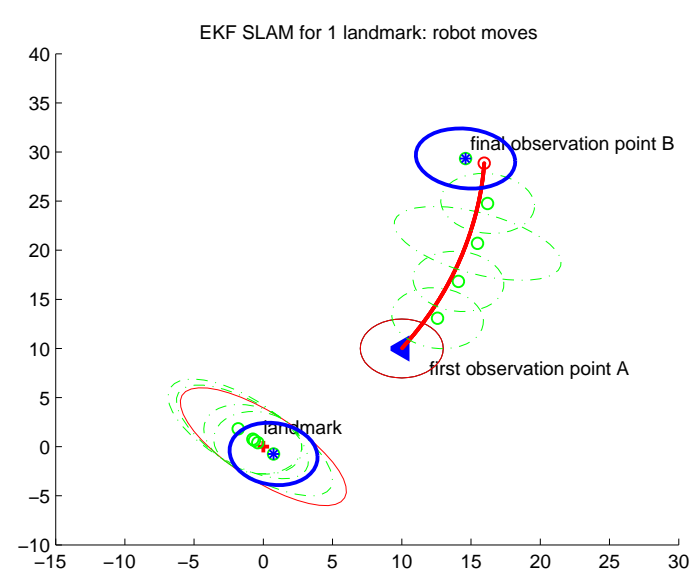

(a) move 500 steps with no control noise - inconsistency

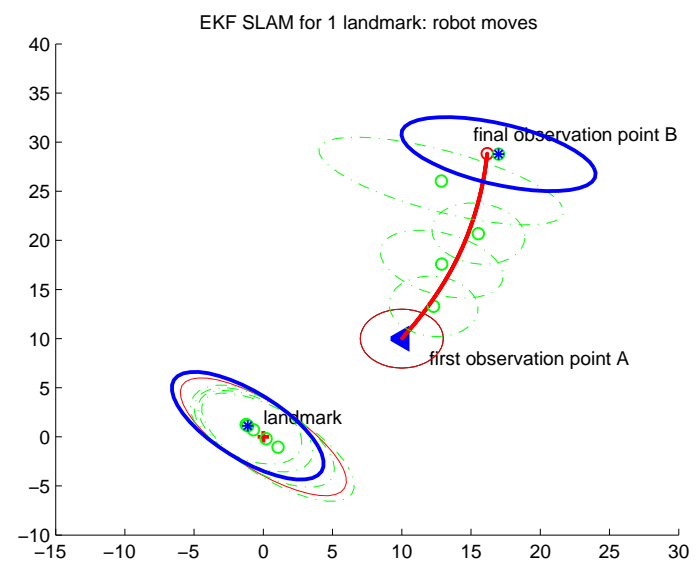

(c) move 500 steps with control noise - inconsistency

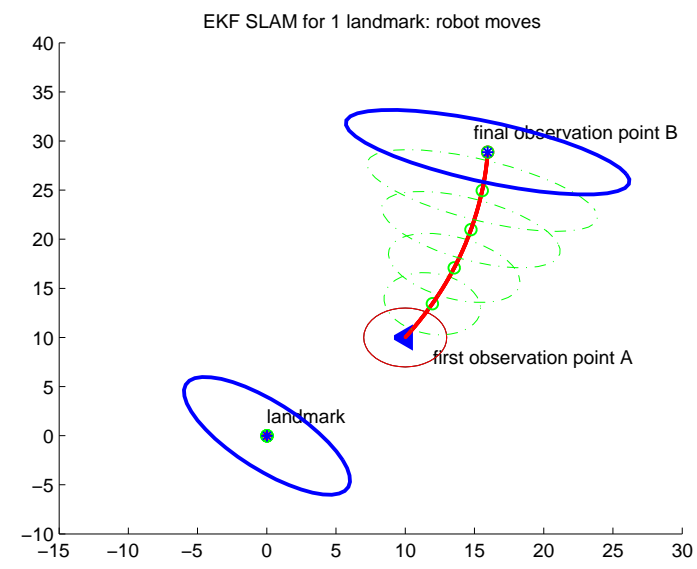

(b) move 500 steps with no control noise - using true Jacobians

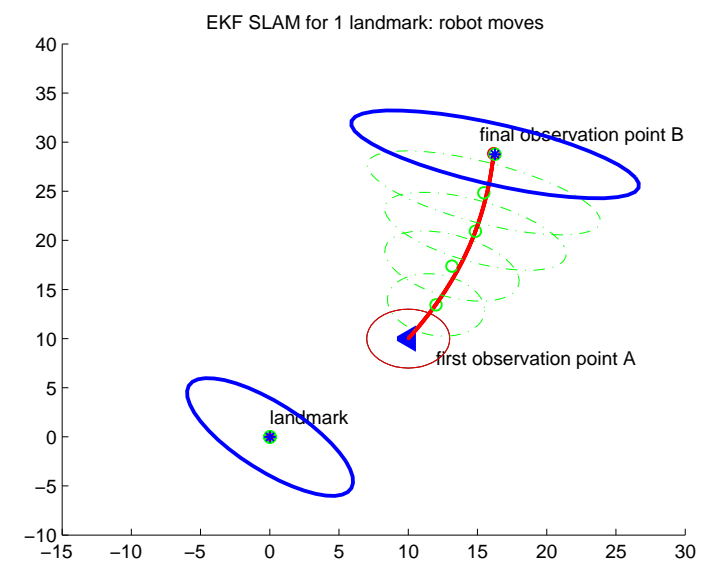

(d) move 500 steps with control noise - using true Jacobians

Fig. 4. The inconsistency of EKF SLAM when the robot moves (see Theorem 4.2): The robot first remains stationary at point A and makes observation $n=10000$ times. Then the robot moves 500 steps to $\mathbf{B}$ and keeps observing the same landmark while moving. The thin/solid ellipses are the uncertainty after the observation at point $\mathbf{A}$, the dashed ellipses are the uncertainties at the intermediate points (every 100 steps), the thick/solid ellipses are the final uncertainties.

In fact, when the robot is stationary, Julier and Uhlmann [7] proved that the state estimate of the robot will remain unchanged if and only if the Jacobians satisfy a particular equality (equation (9) in Theory 1 in [7]). The results presented in Theorems 3.2 and 3.5 of this paper show that if all the Jacobians are evaluated at the true states, then the equation (9) in [7] always holds. Moreover, it is shown that when the robot is in motion, there is another fundamental constraint on the Jacobians (Lemma 3.8) which should be maintained in order to guarantee consistency.

The common idea used in this paper and [7][8][11] is that the consistency of SLAM estimate is evaluated based on the fact "Keep observing new landmarks does not help in reducing the robot pose uncertainty". In [7], the inconsistency is evidenced by the "incorrect update of the mean value of the robot pose estimate". The inconsistency is evidenced by "incorrect reduction of the covariance matrix of the robot pose estimate" in this paper (by deriving the explicit formula) and in [8][11] (by extensive Monte-Carlo simulations).

\section{B. Discussion}

The assumptions made in deriving the results in this paper are: (i) the map consists of point landmarks; (ii) observations consist of ranges and the bearings from the robot to the landmarks; (iii) data association is given; (iv) the process noise and the measurement noise are zero-mean Gaussian; (v) the process noise and sensor errors are all "small" such that EKF is applicable. Note that there is no "linearity" assumption as in [2] and [4]. The results in this paper show that some convergence properties hold if all the Jacobians are evaluated at the true states, but inconsistent estimates can result when the Jacobians are evaluated using the estimated states, as the case in practice. It is also shown that when the robot orientation uncertainty is large, the extent of inconsistency is significant; when the robot orientation uncertainty is small, the extent of inconsistency is insignificant.

It can be expected that similar results hold for other types of landmarks such as lines, corners, etc. although generating appropriate proofs will be more complicated. For example, the inconsistency of EKF SLAM using line features is reported 
in [15]. When the world is observed using range-only or bearing-only sensors, the linearization error will be much larger and the resulting inconsistencies are expected to be more significant. Non-Gaussian control noise and sensor noise may also introduce errors in real SLAM applications, particularly when the robot revisits old landmarks many times.

The insights on the fundamental reasons why EKF SLAM can be inconsistent will help in deriving new variations of EKF SLAM algorithms that minimize the extent of possible inconsistency. For example, if a way to enforce the fundamental constraints of the Jacobians when performing EKF SLAM is found, then the inconsistency of state estimate will be greatly reduced. Since the robot orientation error is one of the main causes of EKF SLAM inconsistency, for large scale SLAM problems, the algorithms that use local submaps (e.g. [16][17][18]), where the robot orientation uncertainties in each local map are kept very small, have the potential to improve consistency.

\section{CONCLUSION}

In this paper, the convergence properties and inconsistency issues of EKF based solution to the nonlinear twodimensional SLAM problem are examined. Explicit formulas for the covariance matrices are provided for several scenarios. It is shown that most of the convergence properties proved by Dissanayake et al. [2] can be generalized to practical nonlinear SLAM problems. It is also proved that inconsistency may occur in EKF SLAM and when the robot orientation uncertainty is large, the estimator inconsistency can result in highly optimistic confidence limits.

The investigation of the limits/lower bounds of the covariance matrices and the consistency analysis for more complicated scenarios (such as closing loops) is the subject of ongoing research. The next step of the research is devoted to develop robust implementation methods of EKF SLAM to minimize possible inconsistency.

\section{APPENDIX A PROOFS OF THE RESUlTS}

Proof of Theorem 3.2: Since the observation noise covariance matrix is $R_{A}$, the information gain from one observation is (see (14)):

$$
\Omega_{n e w}=\nabla H_{A}^{T} R_{A}^{-1} \nabla H_{A} .
$$

For convenience, denote

$$
H_{A}=\left[\begin{array}{ll}
\mathbf{e} & A
\end{array}\right] \text {. }
$$

Thus

$$
\nabla H_{A}=\left[\begin{array}{ll}
-H_{A} & A
\end{array}\right] .
$$

The total information after the $n$ observations is (see the second equation in (13))

$$
\begin{aligned}
= & {\left[\begin{array}{cc}
\Omega_{A_{\text {end }}}^{n} & 0 \\
0 & 0
\end{array}\right]+n\left[\begin{array}{c}
-H_{A}^{T} \\
A^{T}
\end{array}\right] R_{A}^{-1}\left[\begin{array}{ll}
-H_{A} & A
\end{array}\right] } \\
= & {\left[\begin{array}{cc}
\Omega_{0}+n H_{A}^{T} R_{A}^{-1} H_{A} & -n H_{A}^{T} R_{A}^{-1} A \\
-n A^{T} R_{A}^{-1} H_{A} & n A^{T} R_{A}^{-1} A
\end{array}\right] . }
\end{aligned}
$$

By the matrix inversion lemma (equations (95),(97) in Lemma B.1 in Appendix B)

$$
\begin{aligned}
P_{A_{\text {end }}}^{n} & =\left(\Omega_{A_{\text {end }}}^{n}\right)^{-1} \\
& =\left[\begin{array}{cc}
\Omega_{0}^{-1} & \Omega_{0}^{-1} H_{A}^{T} A^{-T} \\
A^{-1} H_{A} \Omega_{0}^{-1} & P_{A_{m}}^{n}
\end{array}\right] \\
& =\left[\begin{array}{cc}
P_{0} & P_{0} H_{A}^{T} A^{-T} \\
A^{-1} H_{A} P_{0} & P_{A_{m}}^{n}
\end{array}\right]
\end{aligned}
$$

where

$$
P_{A_{m}}^{n}=A^{-1} H_{A} P_{0} H_{A}^{T} A^{-T}+\frac{A^{-1} R_{A} A^{-T}}{n} .
$$

Equation (67) is the same as equation (27) because

$$
A^{-1} H_{A}=\left[A^{-1} \mathbf{e} \quad I\right]=A_{\mathbf{e}} .
$$

When $n \rightarrow \infty$, the second item in (68) goes to 0 , so (28) holds. The proof is completed.

Proof of Corollary 3.3: It is clear that the uncertainty of the robot does not change in (27) (will always be $P_{0}$ ). The limit $P_{A_{m}}^{\infty}$ in (29) can be computed further as

$$
\begin{aligned}
P_{A_{m}}^{\infty}= & A_{\mathbf{e}} P_{0} A_{\mathbf{e}}^{T} \\
= & {\left[\begin{array}{ll}
A^{-1} \mathbf{e} & I
\end{array}\right]\left[\begin{array}{cc}
p_{\phi} & \mathbf{p}_{x y \phi}^{T} \\
\mathbf{p}_{x y \phi} & P_{x y}
\end{array}\right]\left[\begin{array}{c}
\mathbf{e}^{T} A^{-T} \\
I
\end{array}\right] } \\
= & P_{x y}+A^{-1} \mathbf{e} p_{\phi} \mathbf{e}^{T} A^{-T} \\
& +A^{-1} \mathbf{e p}_{x y \phi}^{T}+\mathbf{p}_{x y \phi} \mathbf{e}^{T} A^{-T}
\end{aligned}
$$

When $p_{\phi} \rightarrow 0$ (then $\mathbf{p}_{x y \phi} \rightarrow 0$ because $P_{0}$ is positive definite), the limit $P_{A_{m}}^{\infty} \rightarrow P_{x y}$. The proof is completed.

Proof of Lemma 3.8: Since the robot moves from $\mathbf{A}$ to $\mathbf{B}$ following the process model, the Jacobians $\nabla H_{A}$ and $\nabla H_{B}$ are not independent. By (24),

$$
A^{-1}=\left[\begin{array}{rr}
\frac{d x_{A}}{r_{A}} & -d y_{A} \\
\frac{d y_{A}}{r_{A}} & d x_{A}
\end{array}\right], \quad A^{-1} \mathbf{e}=\left[\begin{array}{r}
-d y_{A} \\
d x_{A}
\end{array}\right] .
$$

Similarly,

$$
B^{-1} \mathbf{e}=\left[\begin{array}{r}
-d y_{B} \\
d x_{B}
\end{array}\right] .
$$

Note that the relationship between the positions of point $\mathbf{A}$ and point $\mathbf{B}$ is:

$$
\begin{aligned}
& x_{B}=x_{A}+v T \cos \left(\phi_{A}\right) \\
& y_{B}=y_{A}+v T \sin \left(\phi_{A}\right) .
\end{aligned}
$$

Thus

$$
\begin{aligned}
d x_{B} & =x_{m}-x_{B}=d x_{A}-v T \cos \left(\phi_{A}\right) ; \\
d y_{B} & =y_{m}-y_{B}=d y_{A}-v T \sin \left(\phi_{A}\right) .
\end{aligned}
$$

So

$$
A^{-1} \mathbf{e}-B^{-1} \mathbf{e}=\left[\begin{array}{r}
-v T \sin \left(\phi_{A}\right) \\
v T \cos \left(\phi_{A}\right)
\end{array}\right] .
$$

From (10),

$$
\begin{aligned}
& B_{\mathbf{e}} \nabla f_{\phi X_{r}}^{A}=\left[B^{-1} \mathbf{e} \quad I\right]\left[\left[\begin{array}{cc}
1 & 0 \\
-v T \sin \left(\phi_{A}\right) \\
v T \cos \left(\phi_{A}\right)
\end{array}\right] \quad I\right] \\
& =\left[B^{-1} \mathbf{e}+\left[\begin{array}{r}
-v T \sin \left(\phi_{A}\right) \\
v T \cos \left(\phi_{A}\right)
\end{array}\right] I\right] \\
& =\left[\begin{array}{ll}
A^{-1} \mathbf{e} & I
\end{array}\right] \\
& =A_{\mathbf{e}} \text {. }
\end{aligned}
$$


The proof of the lemma is completed.

Proof of Theorem 3.9: Suppose the robot observed $n$ times ( $n \rightarrow \infty$ will be considered later) the landmark $m$ at point A. Before the robot moves to point $\mathbf{B}$, the covariance matrix is $P_{A_{\text {end }}}^{n}$ given by (27). By the prediction formula (8), the covariance matrix when the robot reaches point $\mathbf{B}$ is

$$
P_{B_{\text {start }}}=\left[\begin{array}{cc}
P_{r r} & P_{r m} \\
P_{m r} & P_{A_{m}}^{n}
\end{array}\right]
$$

where

$$
\begin{aligned}
P_{r r} & =\nabla f_{\phi X_{r}}^{A} P_{0}\left(\nabla f_{\phi X_{r}}^{A}\right)^{T}+\nabla f_{\gamma v}^{A} \Sigma\left(\nabla f_{\gamma v}^{A}\right)^{T} \\
P_{r m} & =\nabla f_{\phi X_{r}}^{A} P_{0} A_{\mathbf{e}}^{T} \\
P_{m r} & =A_{\mathbf{e}} P_{0}\left(\nabla f_{\phi X_{r}}^{A}\right)^{T} \\
P_{A_{m}}^{n} & =A_{\mathbf{e}} P_{0} A_{\mathbf{e}}^{T}+\frac{A^{-1} R_{A} A^{-T}}{n} .
\end{aligned}
$$

Similar to (65), denote

$$
H_{B}=\left[\begin{array}{ll}
\mathbf{e} & B
\end{array}\right]
$$

Thus

$$
\nabla H_{B}=\left[\begin{array}{ll}
-H_{B} & B
\end{array}\right]
$$

The total information after $l$ observations at point $\mathbf{B}$ is

$$
\Omega_{B_{\text {end }}}^{l}=\Omega_{B_{\text {start }}}+l\left[\begin{array}{c}
-H_{B}^{T} \\
B^{T}
\end{array}\right] R_{B}^{-1}\left[\begin{array}{ll}
-H_{B} & B
\end{array}\right]
$$

where $\Omega_{B_{\text {start }}}=P_{B_{\text {start }}}^{-1}$ and $R_{B}$ is the covariance matrix of the observation noise.

Denote

$$
C_{B}=\nabla H_{B}=\left[\begin{array}{ll}
-H_{B} & B
\end{array}\right], \quad D_{B}=\frac{R_{B}}{l} .
$$

Using the matrix inversion lemma (see (99) in Appendix $B$ ), the covariance matrix after the observations at point $\mathbf{B}$ is

$$
\begin{aligned}
& P_{B_{\text {end }}}^{l} \\
= & \left(\Omega_{B_{\text {end }}}^{l}\right)^{-1} \\
= & \Omega_{B_{\text {start }}}^{-1}-\Omega_{B_{\text {start }}}^{-1} C_{B}^{T}\left(D_{C P C}\right)^{-1} C_{B} \Omega_{B_{\text {start }}}^{-1} \\
= & P_{B_{\text {start }}}-P_{B_{\text {start }}} C_{B}^{T}\left(D_{C P C}\right)^{-1} C_{B} P_{B_{\text {start }}}
\end{aligned}
$$

where

$$
D_{C P C}=D_{B}+C_{B} P_{B_{\text {start }}} C_{B}^{T} .
$$

By direct computation,

$$
C_{B} P_{B_{\text {start }}}=\left[\begin{array}{ll}
C_{P 1} & C_{P 2}
\end{array}\right]
$$

where

$$
\begin{aligned}
C_{P 1} & =\Delta_{A B} P_{0}\left(\nabla f_{\phi X_{r}}^{A}\right)^{T}-H_{B} \nabla f_{\gamma v}^{A} \Sigma\left(\nabla f_{\gamma v}^{A}\right)^{T} \\
& =\Delta_{A B} P_{0}\left(\nabla f_{\phi X_{r}}^{A}\right)^{T}-H_{A B} \Sigma\left(\nabla f_{\gamma v}^{A}\right)^{T}, \\
C_{P 2} & =\Delta_{A B} P_{0} A_{\mathbf{e}}^{T}+\frac{1}{n} B A^{-1} R_{A} A^{-T}
\end{aligned}
$$

with

$$
\Delta_{A B}=B A_{\mathbf{e}}-H_{B} \nabla f_{\phi X_{r}}^{A}=B\left(A_{\mathbf{e}}-B_{\mathbf{e}} \nabla f_{\phi X_{r}}^{A}\right)
$$

and $H_{B}$ defined in (74) and $H_{A B}$ defined in (43).

By Lemma 3.8, $\Delta_{A B}=0$, so from (79),

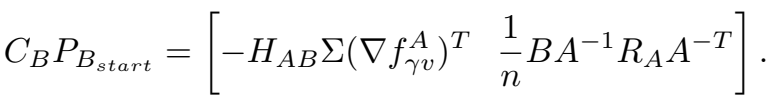

Let $n \rightarrow \infty$, then

$$
C_{B} P_{B_{\text {start }}}=\left[\begin{array}{ll}
-H_{A B} \Sigma\left(\nabla f_{\gamma v}^{A}\right)^{T} & 0
\end{array}\right],
$$

and

$$
D_{B}+C_{B} P_{B_{\text {start }}} C_{B}^{T}=\frac{1}{l} R_{B}+H_{A B} \Sigma H_{A B}^{T} .
$$

So from (77), (78) and let $n \rightarrow \infty$,

$$
\begin{aligned}
& P_{B_{\text {end }}}^{l}=P_{B_{\text {start }}}-\left[\begin{array}{c}
-\nabla f_{\gamma v}^{A} \Sigma H_{A B}^{T} \\
0
\end{array}\right] \\
& \begin{array}{l}
\cdot\left(\frac{1}{l} R_{B}+H_{A B} \Sigma H_{A B}^{T}\right)^{-1} \\
\cdot\left[-H_{A B} \Sigma\left(\nabla f_{\gamma v}^{A}\right)^{T} 0\right]
\end{array} \\
& =P_{B_{\text {start }}}^{0}+\left[\begin{array}{cc}
\Delta_{l} & 0 \\
0 & 0
\end{array}\right]
\end{aligned}
$$

where $P_{B_{\text {start }}}^{0}$ is defined in (40) and

$$
\begin{aligned}
\Delta_{l}= & \nabla f_{\gamma v}^{A} \Sigma\left(\nabla f_{\gamma v}^{A}\right)^{T}-\nabla f_{\gamma v}^{A} \Sigma H_{A B}^{T} \\
& \cdot\left(\frac{1}{l} R_{B}+H_{A B} \Sigma H_{A B}^{T}\right)^{-1} H_{A B} \Sigma\left(\nabla f_{\gamma v}^{A}\right)^{T} \\
= & \nabla f_{\gamma v}^{A}\left[\Sigma-\Sigma H_{A B}^{T}\left(\frac{1}{l} R_{B}+H_{A B} \Sigma H_{A B}^{T}\right)^{-1}\right. \\
& \left.\cdot H_{A B} \Sigma\right]\left(\nabla f_{\gamma v}^{A}\right)^{T} .
\end{aligned}
$$

By matrix inversion lemma (equation (99) in Appendix B),

$$
\begin{aligned}
& \Sigma-\Sigma H_{A B}^{T}\left(\frac{1}{l} R_{B}+H_{A B} \Sigma H_{A B}^{T}\right)^{-1} H_{A B} \Sigma \\
= & {\left[\Sigma^{-1}+l H_{A B}^{T} R_{B}^{-1} H_{A B}\right]^{-1} \geq 0 . }
\end{aligned}
$$

Thus

$$
\Delta_{l}=\nabla f_{\gamma v}^{A} \Sigma_{B}^{l}\left(\nabla f_{\gamma v}^{A}\right)^{T}
$$

with $\Sigma_{B}^{l}$ defined in (42). By (85), (39) holds.

It is easy to see from (42) that if the matrix $H_{A B}^{T} R_{B}^{-1} H_{A B}$ is invertible, then $\Sigma_{B}^{l} \rightarrow 0$ and hence $P_{B}^{l} \rightarrow 0$ as $l \rightarrow \infty$. The proof is completed.

Proof of Theorem 4.1: The initial robot information is $\Omega_{0}$ in (22). The final information after the $n$ observations is

$$
\begin{aligned}
\Omega_{1}= & {\left[\begin{array}{cc}
\Omega_{0} & 0 \\
0 & 0
\end{array}\right]+\sum_{j=1}^{n} \nabla H_{\tilde{A}_{j}}^{T} R_{A}^{-1} \nabla H_{\tilde{A}_{j}} } \\
= & {\left[\begin{array}{ccc}
i_{\phi} & \mathbf{b}^{T} & 0 \\
\mathbf{b} & \Omega_{x y} & 0 \\
0 & 0 & 0
\end{array}\right] } \\
& +\sum_{j=1}^{n}\left[\begin{array}{c}
-\mathbf{e}^{T} \\
-\tilde{A}_{j}^{T} \\
\tilde{A}_{j}^{T}
\end{array}\right] R_{A}^{-1}\left[\begin{array}{lll}
-\mathbf{e} & -\tilde{A}_{j} & \tilde{A}_{j}
\end{array}\right] \\
= & {\left[\begin{array}{cc}
i_{\phi 1} & \mathbf{b}_{1}^{T} \\
\mathbf{b}_{1} & \Omega_{x y 1}
\end{array}\right] }
\end{aligned}
$$

where

$$
\begin{aligned}
i_{\phi 1} & =i_{\phi}+n \mathbf{e}^{T} R_{A}^{-1} \mathbf{e}, \\
\mathbf{b}_{1} & =\left[\begin{array}{c}
\mathbf{b}+\left(\sum_{j=1}^{n} \tilde{A}_{j}^{T}\right) R_{A}^{-1} \mathbf{e} \\
-\left(\sum_{j=1}^{n} \tilde{A}_{j}^{T}\right) R_{A}^{-1} \mathbf{e}
\end{array}\right], \\
\Omega_{x y 1} & =\left[\begin{array}{cc}
\Omega_{x y}+\Omega_{\Sigma_{j}} & -\Omega_{\Sigma_{j}} \\
-\Omega_{\Sigma_{j}} & \Omega_{\Sigma_{j}}
\end{array}\right]
\end{aligned}
$$

with

$$
\Omega_{\Sigma_{j}}=\sum_{j=1}^{n} \tilde{A}_{j}^{T} R_{A}^{-1} \tilde{A}_{j}
$$

Since $\Omega_{x y}$ and $\Omega_{\Sigma_{j}}$ are all positive definite matrices, it can be proved that

$$
\Omega_{x y 1}^{-1}=\left[\begin{array}{cc}
\Omega_{x y}^{-1} & \Omega_{x y}^{-1} \\
\Omega_{x y}^{-1} & \Omega_{x y}^{-1}+\Omega_{\Sigma_{j}}^{-1}
\end{array}\right]
$$


and hence

$$
\begin{aligned}
\mathbf{b}_{1}^{T} \Omega_{x y 1}^{-1} \mathbf{b}_{1}= & \mathbf{b}^{T} \Omega_{x y}^{-1} \mathbf{b}+\mathbf{e}^{T} R_{A}^{-1}\left(\sum_{j=1}^{n} \tilde{A}_{j}\right) \\
& \cdot \Omega_{\Sigma_{j}}^{-1}\left(\sum_{j=1}^{n} \tilde{A}_{j}^{T}\right) R_{A}^{-1} \mathbf{e}
\end{aligned}
$$

Now apply the matrix inversion lemma to $\Omega_{1}$,

$$
\begin{aligned}
P_{1} & =\Omega_{1}^{-1} \\
& =\left[\begin{array}{cc}
\left(i_{\phi 1}-\mathbf{b}_{1}^{T} \Omega_{x y 1}^{-1} \mathbf{b}_{1}\right)^{-1} & * \\
* & *
\end{array}\right] \\
& =\left[\begin{array}{cc}
\left(i_{\phi}-\mathbf{b}^{T} \Omega_{x y}^{-1} \mathbf{b}+w(n, A)\right)^{-1} & * \\
* & *
\end{array}\right]
\end{aligned}
$$

where $*$ stands for a matrix that is not cared about, and $w(n, A)$ is defined in (60).

By the definition (60),

$$
w(n, A)=n \mathbf{e}^{T} W \mathbf{e}
$$

where

$$
\begin{aligned}
W= & R_{A}^{-1}-R_{A}^{-1}\left(\sum_{j=1}^{n} \tilde{A}_{j}\right)\left(n \sum_{j=1}^{n} \tilde{A}_{j}^{T} R_{A}^{-1} \tilde{A}_{j}\right)^{-1} \\
& \cdot\left(\sum_{j=1}^{n} \tilde{A}_{j}^{T}\right) R_{A}^{-1} .
\end{aligned}
$$

Using the inequality

$$
n \sum_{j=1}^{n} \tilde{A}_{j}^{T} R_{A}^{-1} \tilde{A}_{j} \geq\left(\sum_{j=1}^{n} \tilde{A}_{j}^{T}\right) R_{A}^{-1}\left(\sum_{j=1}^{n} \tilde{A}_{j}\right),
$$

it can be shown that $W \geq 0$ and thus

$$
w(n, A) \geq 0 .
$$

So in (91),

$$
\left(i_{\phi}-\mathbf{b}^{T} \Omega_{x y}^{-1} \mathbf{b}+w(n, A)\right)^{-1} \leq\left(i_{\phi}-\mathbf{b}^{T} \Omega_{x y}^{-1} \mathbf{b}\right)^{-1}=p_{\phi} .
$$

This means that the updated robot orientation uncertainty cannot be greater than the initial robot orientation uncertainty.

Furthermore, if matrices $\tilde{A}_{j}, 1 \leq j \leq n$ are all the same, then (93) becomes an equality and

$$
w(n, A)=0
$$

and hence

$$
\left(i_{\phi}-\mathbf{b}^{T} \Omega_{x y}^{-1} \mathbf{b}+w(n, A)\right)^{-1}=p_{\phi} .
$$

However, if matrices $\tilde{A}_{j}, 1 \leq j \leq n$ are different, then

$$
w(n, A)>0
$$

and

$$
\left(i_{\phi}-\mathbf{b}^{T} \Omega_{x y}^{-1} \mathbf{b}+w(n, A)\right)^{-1}<p_{\phi} .
$$

It is obvious that the robot orientation uncertainty cannot be reduced by observing a single new landmark. So this is wrong (inconsistent). In general, if matrices $\tilde{A}_{j}, 1 \leq j \leq n$ are different, then $w(n, A) \rightarrow \infty$ when $n \rightarrow \infty$, thus

$$
\left(i_{\phi}-\mathbf{b}^{T} \Omega_{x y}^{-1} \mathbf{b}+w(n, A)\right)^{-1} \rightarrow 0 .
$$

This means that the uncertainty of the robot orientation will decrease to 0 after many observations. The proof is completed.

Proof of Theorem 4.2: The proof is only given for the simple case when there is no control noise, i.e. $\Sigma=0$. In this case, if $\Delta_{A B}=0$, then $C_{P 1}=0$ in (79); if $\Delta_{A B} \neq 0$, then
$C_{P 1}=\Delta_{A B} P_{0}\left(\nabla f_{\phi X_{r}}^{A}\right)^{T} \neq 0$. Now by (77) and (79), the upper left submatrix of $P_{B_{\text {end }}}^{l}$ is

$$
\begin{aligned}
& \nabla f_{\phi X_{r}}^{A} P_{0}\left(\nabla f_{\phi X_{r}}^{A}\right)^{T}-C_{P 1}^{T}\left(D_{C P C}\right)^{-1} C_{P 1} \\
\leq & \nabla f_{\phi X_{r}}^{A} P_{0}\left(\nabla f_{\phi X_{r}}^{A}\right)^{T} .
\end{aligned}
$$

This violates the lower bound proved in Theorem 3.9.

\section{APPENDIX B \\ MATRIX INVERSION LEMMA}

The following matrix inversion lemma is used frequently in the proofs of the results in this paper. It can be found in many textbooks about matrices or Kalman Filter (e.g. [19]).

Lemma B.1: [19] Suppose that the partitioned matrix

$$
M=\left[\begin{array}{cc}
A & B \\
C & D
\end{array}\right]
$$

is invertible and that the inverse is conformably partitioned as

$$
M^{-1}=\left[\begin{array}{cc}
X & Y \\
U & V
\end{array}\right],
$$

where $A, D, X$ and $V$ are square matrices. If $A$ is invertible, then

$$
\begin{aligned}
X & =A^{-1}+A^{-1} B\left(D-C A^{-1} B\right)^{-1} C A^{-1}, \\
Y & =-A^{-1} B\left(D-C A^{-1} B\right)^{-1} \\
U & =-\left(D-C A^{-1} B\right)^{-1} C A^{-1} \\
V & =\left(D-C A^{-1} B\right)^{-1} .
\end{aligned}
$$

If $D$ is invertible, then

$$
\begin{aligned}
& X=\left(A-B D^{-1} C\right)^{-1} \\
& Y=-\left(A-B D^{-1} C\right)^{-1} B D^{-1} \\
& U=-D^{-1} C\left(A-B D^{-1} C\right)^{-1} \\
& V=D^{-1}+D^{-1} C\left(A-B D^{-1} C\right)^{-1} B D^{-1} .
\end{aligned}
$$

Thus if both $A$ and $D$ are invertible,

$$
\left(A-B D^{-1} C\right)^{-1}=A^{-1}+A^{-1} B\left(D-C A^{-1} B\right)^{-1} C A^{-1} \text {. }
$$

When $B=C^{T}$, equation (98) can be written as (substituting $D$ by $-D$ )

$$
\left(A+C^{T} D^{-1} C\right)^{-1}=A^{-1}-A^{-1} C^{T}\left(D+C A^{-1} C^{T}\right)^{-1} C A^{-1} .
$$

\section{ACKNOWLEDGMENT}

This work is supported by the ARC Centre of Excellence programme, funded by the Australian Research Council (ARC) and the New South Wales State Government. The authors would also like to thank Tim Bailey and Zhan Wang for helpful discussions.

\section{REFERENCES}

[1] U. Frese, P. Larsson, T. Duckett, "A multilevel relaxation algorithm for simultaneous localization and mapping," IEEE Transactions on Robotics, vol. 21 (2), pp. 196- 207, 2005.

[2] G. Dissanayake, P. Newman, S. Clark, H. Durrant-Whyte, and M. Csorba, "A solution to the simultaneous localization and map building (SLAM) problem," IEEE Trans. on Robotics and Automation, vol. 17, pp. 229-241, 2001.

[3] J. W. Fenwick, P. M. Newman and J. J. Leonard, "Cooperative concurrent mapping and localization", Proceeding of the IEEE Conference on Robotics and Automation, Washington D.C. May 2002, pp. 1810-1817. 
[4] S. J. Kim, Efficient Simultaneous Localization and Mapping Algorithms using Submap Networks, PhD thesis, Dept. of Ocean Engineering, Massachusetts Institute of Technology, 2004.

[5] P.W. Gibbens, G. Dissanayake, and H. Durrant-Whyte, "A closed form solution to the single degree of freedom simultaneous localization and map building (SLAM) problem," Proceeding of the 39th IEEE Conferences on Decision and Control, Sydney, Australia, December 2000, pp. 191-196.

[6] P. M. Newman, On the Structure and Solution of the Simultaneous Localization and Map Building Problem, PhD thesis, Australian Centre of Field Robotics, University of Sydney, Sydney, 2000. Available online http://www.robots.ox.ac.uk/ pnewman/

[7] S. J. Julier and J. K. Uhlmann, "A counter example for the theory of simultaneous localization and map building", Proceeding of the IEEE Conference on Robotics and Automation, Seoul, Korea, May 21-26, 2001, pp. 4238-4243.

[8] J.A. Castellanos, J. Neira and J.D. Tardos. "Limits to the consistency of EKF-based SLAM". 5th IFAC Symp. on Intelligent Autonomous Vehicles, IAV'04, Lisbon, Portugal, July 2004. available online http://webdiis.unizar.es/\%7Ejdtardos/publications.html

[9] U. Frese. "A discussion of simultaneous localization and mapping". Autonomous Robots 20, 25-42, 2006.

[10] A. Martinell, N. Tomatis, and R. Siegwart, "Some results on SLAM and the closing the loop problem". In Proceedings of the IEEE/RSJ Intenational Conference on Intelligent Robots and Systems, IROS, Edmonton, Canada, 2005, pp. 334-339.

[11] T. Bailey, J. Nieto, J. Guivant, M. Stevens and E. Nebot, "Consistency of the EKF-SLAM algorithm", In Proceedings of the 2006 IEEE/RSJ International Conference on Intelligent Robots and Systems, October 9 15, 2006, Beijing, China, pp. 3562-3568.

[12] S. Thrun, W. Burgard, and D. Fox, Probabilistic Robotics, The MIT Press, 2005.

[13] H. Durrant-Whyte and T. Bailey. "Simultanouse localization and mapping: Part I". IEEE Robotics \& Automation Magazine, 99-108, June 2006.

[14] M. Csorba, Simultaneous Localisation and Map Building, Ph.D dissertation, Univ. Oxford, 1997.

[15] D. Rodriguez-Losada, F. Matia, A. Jimenez, and R. Galan, "Consistency improvement for SLAM-EKF for indoor environments". Proceeding of the IEEE Conference on Robotics and Automation, Orlando, Florida, May 2006, pp. 418-423.

[16] J. D. Tardos, J. Neira, P. M. Newman and J. J. Leonard, "Robust mapping and localization in indoor environments using sonar data", The International Journal of Robotics Research, Vol. 21, No. 4, April 2002, pp. 311-330.

[17] S. B. Williams, Efficient Solutions to Autonomous Mapping and Navigation Problems, $\mathrm{PhD}$ thesis, Australian Centre of Field Robotics, University of Sydney, Sydney, 2001. available online http://www.acfr.usyd.edu.au/

[18] S. Huang, Z. Wang and G. Dissanayake, "Mapping large scale environments using relative position information among landmarks", 2006 IEEE International Conference on Robotics and Automation (ICRA), Orlando, Florida, USA, May 15-19, 2006, 2297-2302.

[19] F. Zhang, Matrix Theory: Basic Results and Techniques, Springer-Verlag. 1999. 